
\title{
28 Research Suare \\ Transient Cell Cycle Induction in Cardiomyocytes to Treat Ischemic Heart Failure
}

\section{Riham Abouleisa}

University of Louisville

Qinghui Ou

University of Louisville

Xian-Liang Tang

University of Louisville

Mitesh Solanki

University of Louisville

Yiru Guo

University of Louisville

Yibing Nong

University of Louisville https://orcid.org/0000-0001-7934-7821

Lindsey Mcnally

University of Louisville

Pawel Lorkiewicz

University of Louisville

Kamal Kassem

University of Louisville

Brooke Ahern

University of Kentucky

Krishna Choudhary

Gladstone Institute https://orcid.org/0000-0002-7966-1527

\section{Reuben Thomas}

Gladstone Institute

\section{Yu Huang}

Gladstone Institute

Hamzah Juhardeen

Alfaisal University

Aisha Siddique

Alfaisal University

Zainab Ifthikar

Alfaisal University

Abou-Bakr Salama 
University of Louisville

\section{Sally Hammad}

University of Louisville https://orcid.org/0000-0003-3887-4398

\section{Ayman Elbaz}

University of Louisville

Kathryn Ivey

Tenaya Therapeutics

Jonathan Satin

University of Kentucky

\section{Bradford Hill}

University of Louisville

\section{Deepak Srivastava}

Gladstone Institute

\section{Roberto Bolli}

University of Louisville

Tamer Mohamed ( $\nabla$ tamer.mohamed@louisville.edu )

University of Louisville

\section{Article}

Keywords: myocardial infarction (MI), regenerative capacity

Posted Date: December 28th, 2020

DOI: https://doi.org/10.21203/rs.3.rs-122026/v1

License: (c) (i) This work is licensed under a Creative Commons Attribution 4.0 International License.

Read Full License 


\section{Abstract}

The regenerative capacity of the heart after myocardial infarction (MI) is limited. Our previous study showed that ectopic introduction of Cdk1/CyclinB1 and Cdk4/CyclinD1 complexes (4F) promotes cardiomyocyte proliferation in $15-20 \%$ of infected cardiomyocytes in vitro and in vivo and improves cardiac function after MI. Here, we aim to identify the necessary reprogramming stages during the forced cardiomyocyte proliferation with $4 \mathrm{~F}$ on a single cell basis. Also, we aim to start the first preclinical testing to introduce $4 \mathrm{~F}$ gene therapy as a candidate for the treatment of ischemia-induced heart failure. Temporal bulk and single-cell RNAseq and further biochemical validations of mature hiPS-CMs treated with either LacZ or $4 \mathrm{~F}$ adenoviruses revealed full cell cycle reprogramming in $15 \%$ of the cardiomyocyte population after $48 \mathrm{~h}$ post-infection with $4 \mathrm{~F}$, which was associated with sarcomere disassembly and metabolic reprogramming. Transient overexpression of 4F, specifically in cardiomyocytes, was achieved using a polycistronic non-integrating lentivirus (NIL) encoding the 4F; each is driven by a TNNT2 promoter (TNNT2-4F-NIL). TNNT2-4F-NIL or control virus was injected intramyocardially one week after MI in rats or pigs. TNNT2-4F-NIL treated animals showed significant improvement in left ventricular ejection fraction and scar size compared with the control virus treated animals four weeks post-injection.

In conclusion, the present study provides a mechanistic demonstration of the process of forced cardiomyocyte proliferation and advances the clinical feasibility of this approach by minimizing the oncogenic potential of the cell cycle factors using a novel transient and cardiomyocyte-specific viral construct.

\section{Introduction}

The mammalian cell cycle involves numerous feedback loops that either permit or prevent cell division depending on the cell type ${ }^{1,2}$. Although fetal myocytes proliferate to achieve cardiac growth and tissuespecific stem cells undergo cytokinesis postnatally, differentiated cells typically become post-mitotic and permanently exit the cell cycle ${ }^{3}$. As a result, the regenerative capacity of most organs, including the heart, is limited. The ability to control proliferation in the postnatal heart would represent a powerful approach to promote cardiac repair after infarction.

Recently, we took a combinatorial approach to screen for factors and conditions that could recapitulate the fetal state of cardiomyocyte division. We found that ectopic introduction of the Cdk1/CyclinB1 and the Cdk4/CyclinD1 complexes (4F, i.e., four factors) promoted cell division in at least $15 \%$ of mouse and human cardiomyocytes in vitro ${ }^{4}$. Rigorous assessment of cardiomyocyte cytokinesis in vivo using the Cre-recombinase dependent Mosaic Analysis with Double Markers (MADM) ${ }^{5}$ lineage tracing system revealed similar efficiency in mouse hearts, leading to cardiac regeneration upon delivery of the $4 \mathrm{~F}$ one week after myocardial infarction ${ }^{4}$. Moreover, in vitro and in vivo results show that myocytes undergo only one round of division after transduction with these cell cycle factors because the overexpression of the $4 \mathrm{~F}$ in cardiomyocytes is self-limiting through proteasome-mediated degradation of the protein products ${ }^{4}$. Interestingly, a recent study showed that AAV-mediated expression of microRNA-199a in infarcted pig 
hearts could initially stimulate cardiac repair through induction of cardiomyocyte proliferation; however, subsequent persistent and uncontrolled expression of the microRNA resulted in sudden arrhythmic death of most of the treated pigs ${ }^{6}$. Our approach is currently one of the most robust methods for inducing cardiomyocyte proliferation; however, the timing, dosage, and specificity of this therapy's expression must be tightly controlled. Therefore, there is a need to transiently and specifically express $4 \mathrm{~F}$ in cardiomyocytes to induce one cycle of proliferation to avoid any adverse effects in the heart and other tissues if escaped.

Uncertainty regarding the mechanisms underlying the functional improvement observed with cell-based therapies is a significant limitation of these approaches and led to skepticism about their clinical applicability (reviewed in ${ }^{7,8}$ ). Therefore, prior to starting any translational efforts, we first sought to gain mechanistic insights during the cell cycle reprogramming at a single cell transcriptomics level in a temporal manner following overexpression of the $4 \mathrm{~F}$ in cardiomyocytes. We were able to identify the main reprogramming steps needed for the cardiomyocytes to complete the cell cycle. These findings provide an essential foundation that enables one to ascribe subsequent cardiac functional improvements to the generation of new cardiomyocytes. Then, we provided the first proof-of-concept evidence for this approach's clinical applicability by minimizing any oncogenic potential of the cell cycle factors using a novel transient and cardiomyocyte-specific viral construct in rat and pig models of heart failure.

\section{Results}

\section{Temporal bulk and single-cell RNAseq of mature hiPS-CMs reveals that cell cycle reprogramming is associated with sarcomere disassembly and metabolic reprogramming during forced cell cycle induction}

Previously, we reported that $4 \mathrm{~F}$ induces at least $15 \%$ of mouse and human cardiomyocytes in vitro and in vivo to undergo proliferation within the first $48 \mathrm{~h}$ post infection ${ }^{4}$. This high percentage of proliferating cells within the bulk population provided confidence that temporal bulk RNAseq could identify the significant transcriptional reprogramming events during the progress of cardiomyocyte proliferation at a transcriptional level. Therefore, to investigate the transcriptional changes during cell cycle progression, we conducted temporal bulk RNAseq on 60-day-old mature hiPS-CMs treated with either LacZ (control) or $4 \mathrm{~F}$ adenovirus for 24,48 , or $72 \mathrm{~h}$. Overexpression of the $4 \mathrm{~F}$ induced several transcriptomic changes starting at $48 \mathrm{~h}$ post-infection when proliferation is prominent, as evidenced by the PCA and the volcano plots of the global gene expression (Fig. 1a-b \& Source Data 1). The gene ontology (GO) analysis of the differentially expressed genes between the 4F-48-h group and the LacZ group showed significant upregulation of genes involved in cell cycle progression (Fig. 1c \& Source Data 2). Furthermore, there are time-dependent upregulation of cell cycle activation genes and downregulation of the cell cycle inhibitory genes (Fig. 1d \& Source Data 1).

This cell cycle reprogramming was associated with downregulation of the sarcomeric and contractile genes, which peaks at $48 \mathrm{~h}$ post-infection and starts to decline back at $72 \mathrm{~h}$ (Fig. 2a-b \& Source Data 1\&2). These data suggest that cardiomyocytes need to withdraw from their primary function of 
contraction during the cell cycle to enter the cell cycle. These transcriptomics changes were functionally confirmed by using time-lapse impedance contractility tracing. We found that cardiomyocyte contractile force declined significantly during proliferation (48 $\mathrm{h}$ post-infection), which coincided with the appearance of arrhythmic episodes; normal contractile force and rhythm returned to baseline levels $84 \mathrm{~h}$ post-viral infection (Fig. 2c). Furthermore, during the G2/M phase, especially during anaphase or cytokinesis, the sarcomeric structures were disrupted, as shown by troponin-T Immunostaining of the sarcomeres (Fig. 2d). The impedance-based force measurements indicate the contractile force generated by the monolayer sheet of cardiomyocytes, which could have a mixed-signal from proliferating and nonproliferating cardiomyocytes. Therefore, we investigated the sarcomeric disassembly implication on the electrophysiological properties and ion current in cardiomyocytes during proliferation. The decrease of the Scn5a transcript suggested a possible change in resulting $I_{N a}$, but the transient nature of the scn5a decrease along with the knowledge that transcript levels do not necessarily predict levels of functioning protein motivated us to assess $I_{\mathrm{Na}}$. Mean $I_{\mathrm{Na}}$ density tended to increase compared to LacZ (Extended data Fig. 1a), but the difference between lacZ and the $4 \mathrm{~F} 48$ or $72 \mathrm{~h}$ time points was not significantly different. Cell capacitance was also not different (Extended data Fig. 1b). We also assessed the voltagedependence of steady-state inactivation because there is a development shift of the inactivation midpoint $\left(V_{0.5}\right)$ whereby $V_{0.5}$ becomes more negative with embryonic heart maturation ${ }^{9}$. As with current density, there was no significant difference caused by $4 \mathrm{~F}$ (Extended data Fig. 1c), though there is a trend towards a more negative $\mathrm{V}_{0.5}$ (Extended data Fig. $1 \mathrm{~d}$ ). These data suggest that $4 \mathrm{~F}$ does not necessarily reduce excitability, and there is an unexpected tendency for larger current with more mature properties in cells treated with $4 \mathrm{~F}$ for 48 and $72 \mathrm{~h}$ compared to control cardiomyocytes.

To further investigate in detail the transcriptional modifications during cardiomyocyte proliferation at a single-cell level, we conducted a temporal single-cell RNAseq of 60-day-old mature hiPS-CMs infected with either LacZ (control) or $4 \mathrm{~F}$ adenovirus for 24,48 , and $72 \mathrm{~h}$. Gene expression data were collected from $\sim 7000$ cells/condition as summarized UMAP blots (Fig. 3a). All cells were positive for cardiac markers (TNNT2, TNNC1, and MYH7) (Fig. 3b). Interestingly, a unique cell population that appears $48 \mathrm{~h}$ after infection with the $4 \mathrm{~F}$ expressed high levels of mitosis/cytokinesis genes (Ki67, Aurora Kinase A and B, E2F1, CDC20, ANLN, TK1, CCNA2, PLK1, and PCNA); this population was identified as the mitotic population (Fig. $3 \mathrm{C}$ ). Consistent with our published data ${ }^{4}$, this population represents $\sim 15 \%$ of the total cardiomyocyte population expressing 4F (1143/7269 cells) (Fig. 3d \& Source Data 3). Trajectory analysis using the MONOCLE algorithm confirmed that the $24 \mathrm{~h}$ unique subpopulation develops into the premitotic $48 \mathrm{~h}$ subpopulation, which develops into the $48 \mathrm{~h}$ mitotic subpopulation, then returns to a quiescent state after $72 \mathrm{~h}$ (Fig. 3e). Comparison of gene expression between pre-mitotic and mitotic subpopulations from the $4 \mathrm{~F} 48 \mathrm{~h}$ samples revealed upregulation of the cell cycle genes and downregulation of sarcomeric gene expression in the mitotic subpopulation (Extended data Fig. 2a \& Source Data 4), which supports our findings from the bulk RNAseq. Compared with the quiescent population from the same sample (48 $\mathrm{h}$ post-infection), this unique population of proliferating cardiomyocytes shows significant upregulation of the cell cycle program and significant downregulation of mitochondrial electron transport chain genes (Extended data Fig. 2b \& Source Data 5). 
Consistent with these transcriptomic changes, hiPS-CMs had markedly lower oxygen consumption and extracellular acidification rates $48 \mathrm{~h}$ after $4 \mathrm{~F}$ infection, suggesting lower oxidative phosphorylation and glycolysis in proliferating myocytes (Extended Data Fig. 3a). Furthermore, in 4F-overexpressing hiPS-CMs, stable isotope tracing experiments using ${ }^{13} \mathrm{C}_{6}$-glucose demonstrate significantly higher enrichment of ${ }^{13} \mathrm{C}$ in intermediates or end products in NAD synthesis $\left(\mathrm{NAD}^{+}\right)$, the hexosamine biosynthetic pathway (UDPHexNAc), phosphatidylethanolamine synthesis (CDP-ethanolamine), and pyrimidine nucleobase biosynthesis (uracil) (Extended Data Fig. 3b). These data indicate that proliferating cardiomyocytes diminish catabolic pathway activity and augment biosynthetic pathway activity before or during cell cycle progression.

\section{Transient expression of the 4F using modified RNA showed low infection efficiency}

The insights gained into 4F-mediated cardiomyocyte cell cycle reprogramming encouraged us to standardize the approach to treat heart failure. Our previous studies using adenoviruses provided proof of principle for the efficacy of our approach; ${ }^{4}$ however, the use of adenovirus is not clinically applicable because of the high prevalence of immune response in humans. From our work with adenovirus, we noticed that most of the cardiomyocyte proliferation events occur within the first $48-72 \mathrm{~h}$ after introducing the virus, after which cardiomyocytes shut down the protein expression of the $4 \mathrm{~F}$ through activation of the proteasome system ${ }^{4}$. However, this was not the case in other cell types, such as in neurons, where the expression and proliferation capacity last for over seven days (data not shown as it is out of the context of the manuscript). Furthermore, the induction of long-term cell cycle activity in the heart may become oncogenic and have deleterious effects on the heart ${ }^{6}$. Therefore, we tested the transient expression of the 4F using modified RNA (modRNA) delivery and its ability to induce cardiomyocyte proliferation in vivo using the recently optimized delivery method for delivering modRNA to the heart using citrate sucrose buffer ${ }^{10}$. modRNAs were injected into the myocardium of C57BL/ 6 mice in citrate sucrose buffer; then, we assessed the cardiomyocyte expression of nuclear-GFP, CDK1, CCNB, CDK4, and CCND at $48 \mathrm{~h}$ after injection (Extended Data Fig. 4a-d). The infection efficiency was very low $(<0.01 \%)$ (Extended Data Fig. 4d). To obtain proof-of-principle for the efficacy of the transient expression approach in inducing proper cardiomyocyte cytokinesis, we injected the 4F+GFP modRNAs into the heart of MADM lineage-tracing transgenic mice crossed with tamoxifen-induced alpha-MHC-Cre. Knowing that only a few cells would be informative given the small number of recombined cells expected and the low infection efficiency of the modRNA, we identified the site of injection by the nuclear GFP expression. We found significant induction of phospho-histone $\mathrm{H} 3(\mathrm{pHH} 3)$ in cardiomyocytes at the site of the $4 \mathrm{~F}$ injection compared to control (Extended Data Fig. 4e), suggesting that the modRNAs can induce cell division in vivo. Also, we observed very few MADM recombination events that led to single-colored cells (10-15/heart) that co-localized with pHH3 positive nuclei, suggesting true cytokinesis in this setting (Extended Data Fig. 4f). These data are suggestive that transient expression of the $4 \mathrm{~F}$ may be sufficient to induce cell division but will require more efficient delivery and multiple injections as the infection efficiency and the spread of the modRNA at the injection site is very limited. 


\section{Characterization of cardiomyocyte-specific non-integrating lentivirus system for gene therapy}

The low in vivo infection efficiency of modRNA motivated us to develop and optimize an alternate strategy for $4 \mathrm{~F}$ delivery and transient expression using a non-integrating lentivirus (NIL). NIL is known for its high infection efficiency and transient expression of the encoded protein, which is limited to 2-3 days, followed by a significant decline in expression ${ }^{10-13}$. Several ongoing clinical trials are aiming to treat various diseases with the lentivirus gene therapy approach ${ }^{14}$. This rapid degradation kinetics makes NIL the optimal delivery vehicle for the 4F. Additionally, when using NIL, it is important to target only cardiomyocytes by controlling the 4F expression with the cardiac-specific troponin-T promoter (TNNT2), which we previously optimized ${ }^{15}$.

First, to assess the in vitro kinetics of the gene expression using the proposed viruses, we compared the ability of the CMV early enhancer/chicken beta-actin (CAG) promoter and the TNNT2 promoter to drive the expression of green fluorescent protein (GFP) within the context of integrating lentivirus (I.L.) and NIL over days in hiPS-CMs. hiPS-CMs infected with CAG-GFP-IL and CAG-GFP-NIL showed high infection efficiency and gene expression as early as $48 \mathrm{~h}$ post-infection. In contrast, hiPS-CMs infected with TNNT2-GFP-IL and TNNT2-GFP-NIL did not show high expression until $96 \mathrm{~h}$ post-infection, indicating the slower kinetics of the TNNT2 promoter compared to the CAG promoter. However, CAG-GFP-NIL or TNNT2GFP-NIL kept the expression for only $72 \mathrm{~h}$ post-peak, consistent with previous reports ${ }^{10-13}$ (Extended Data Fig. 5a-c). Then, to investigate the specificity of the TNNT2 promoter to drive the protein expression only in cardiomyocytes, we infected non-cardiomyocytes cells (kidney cells, HEK293), mouse coronary artery endothelial cells (MCAEC), cardiac fibroblasts, and muscle cells ( $\mathrm{H} 9 \mathrm{C} 2$ and $\mathrm{C} 2 \mathrm{C} 12$ myoblasts) with TNNT2-GFP-IL or CAG-GFP-IL (as a control). CAG-GFP-IL resulted in GFP expression over time in all cell types tested at 2, 4, 8 days post-infection; in contrast, TNNT2-GFP-IL did not show any significant GFP expression in any tested cell types (except the cardiomyocytes) up to eight days post-infection. These data suggest that the TNNT2-promoter drives the gene expression specifically in cardiomyocytes (Extended Data Fig. 5d-e).

\section{Polycistronic TNNT2-4F-NIL induced cardiomyocyte proliferation in vitro and in vivo}

Each cell cycle factor of the 4F was cloned into a lentivirus backbone under TNNT2 promoter (4F separate lentiviruses); also, all $4 \mathrm{~F}$ were cloned in one polycistronic lentivirus backbone with each factor is driven by a TNNT2 promoter (4F polycistronic lentivirus) (Extended Data Fig. 6a). First, we assessed each cell cycle factor's protein expression four days post-infection in hiPS-CMs using western blot. TNNT24Fpolycistronic-NIL was significantly more efficient in inducing higher protein expression of all the cell cycle factors compared to the TNNT2-4Fseparate-NIL (Extended Data Fig. 6b-c). Furthermore, TNNT24Fpolycistronic-NIL showed $80-100 \%$ infection efficiency as assessed by immunofluorescence (Extended Data Fig. 6d-e). Four days post-infection with TNNT2-4Fpolycistronic-NIL, we found that $15-20 \%$ of the cardiomyocytes were positive for 5-ethynyl-2'-deoxyuridine (EDU), which marks new DNA synthesis and histone $\mathrm{H} 3$ phosphorylation (PHH3), which marks cells in the G2/M phase (Fig. 4a-b). Furthermore, the total cell number was increased by $20-30 \%$ (Fig. 4b). Assessments 10 days post-infection showed that 
cell number increase and EDU labeling for the divided nuclei persisted; however, the PHH3 was abolished, indicating the transient nature of the cell cycle induction in the cardiomyocyte and the likelihood that TNNT2-4Fpolycistronic-NIL induced only one cycle of proliferation.

To test the efficacy of the TNNT2-4Fpolycistronic-NIL in inducing cardiomyocyte proliferation in vivo, we used a cardiomyocyte cytokinesis lineage-tracing animal model (inducible a-MHC-Cre::MADM-lineagetracing ${ }^{4,5}, 16,17$. In these lineage-tracing mice, cardiomyocytes that undergo cytokinesis produce daughter cells that are either red, green, yellow (red+green), or colorless, based on allelic recombination of fluorescent reporters; if the cardiomyocytes fail to divide, they will remain double-colored (i.e., yellow), or colorless if no recombination occurs. Thus, the presence of single-colored red or green cells definitively indicates cells that have undergone cell division, although dividing cells are underrepresented by singlecolored cells because double-colored (yellow) or colorless cells also could have divided. Animals were subjected to a 60-min occlusion of the left anterior descending artery followed by reperfusion, then oneweek later, TNNT2-4Fpolycistronic-NIL or LacZ-NIL (control) is injected intramyocardially (Fig. 4c). Tamoxifen injection was carried out as described in ${ }^{4}$, starting $48 \mathrm{~h}$ after the virus injection for three days to initiate recombination events. Mice were sacrificed one week after the viral injections, and hearts were sectioned to enumerate the cytokinesis events. All surgeries, imaging, and microscopy analyses were blinded about treatment, and animals were decoded after all data were analyzed. The analysis was done on ten different sections from each heart across the whole myocardium. After intramyocardial injection of TNNT2-4Fpolycistronic-NIL, it showed at least $15 \%$ of the recombinant cardiomyocytes were singlecolored, compared to $<1 \%$ in hearts injected with control virus (Fig. $4 \mathrm{~d}-\mathrm{e}$ ).

\section{TNNT2-4Fpolycistronic-NIL improves cardiac function in a rat model of heart failure}

Before starting in vivo functional studies, we sought to validate that TNNT2-NIL drives cardiomyocytespecific expression of the 4F in vivo. Therefore, TNNT2-4Fpolycistronic-NIL or GFP-NIL control virus were injected intramyocardially, and the rats were sacrificed one-week post-injection. Western blotting and Immunostaining confirmed the expression of $4 \mathrm{~F}$ in the rat hearts six days after injection (Extended Data Fig. 7a-d). Furthermore, RNA expressions of the overexpressed human CDK1, CDK4, CCNB, and CCND in rat hearts were only detected in the heart and not in the other organs six days post-viral injection (Extended Data Fig. 8a).

Then we started to test the effects of TNNT2-4Fpolycistronic-NIL on cardiac function following cardiac damage in vivo. Rats were subjected to a $2 \mathrm{~h}$ coronary occlusion followed by reperfusion. One week later, TNNT2-4Fpolycistronic-NIL or control TNNT2-GFP-NIL was injected into the peri-infarct region of the heart. Rats were followed for four weeks and then sacrificed, and the cardiac tissue was processed and analyzed (Fig. 5a). Gross assessment of the heart weight showed that HW/BW was significantly decreased in the hearts treated with TNNT2-4Fpolycistronic-NIL compared to the control virus treated hearts (Fig. 5b). TNNT2-4Fpolycistronic-NIL-treated groups exhibited a significant increase in left ventricular ejection fraction four weeks post-viral injection compared to the control group, as assessed by blinded echocardiography (Fig. 5c). Consistently with the improvement in cardiac function, histological 
analyses of the hearts revealed an approximately $30 \%$ reduction in the scar size in hearts treated with TNNT2-4Fpolycistronic-NIL compared to control hearts (Fig. 5d). Interestingly, assessment of cell size at the border and remote zones showed a significant reduction in the cardiomyocyte cross-sectional area (Fig. 5e-f). As the virus was injected in the border zone, so the reduction in cardiomyocyte cross-sectional area could be due to the induction of proliferation; however, the reduction in cardiomyocyte crosssectional area at the remote zone could be due to improvement of the overall functionality of the heart and the protection from progression towards dilatation after treatment with TNNT2-4Fpolycistronic-NIL.

\section{Double reporter to permanently label cardiomyocytes activation of aurora kinase B in vivo in large animals}

To track mitotic events in vivo in large animals, we developed a new reporter system based on the Aurora kinase B (AurKB) promoter region activation. AurKB is one of the central protein kinases that ensure the proper execution and fidelity of mitosis and is expressed only for a short time during the cytokinesis process, localizing to the central spindle during anaphase and in the midbody during cytokinesis ${ }^{18}$. It has been considered as a putative marker for mitosis in several cell types, including cardiomyocytes ${ }^{19-21}$. A recent study demonstrated that AurKB correct positioning to the midbody in cardiomyocytes during mitosis is positively correlated with cytokinesis and that $70 \%$ of the neonatal cardiomyocytes that express AurKB undergo complete cytokinesis with correct midbody positioning ${ }^{20}$. To develop this reporter, we used the previously well-characterized $1.8 \mathrm{~kb}$ promoter region of the human AurKB gene, which is highly conserved between species ${ }^{22}$, and cloned it into a lentivirus to drive the expression of GFP protein (Extended Data Fig. 9a). We will refer to this reporter as the AurKB-GFP reporter throughout the manuscript. We generated and validated this reporter to detect mitosis/cytokinesis events in proliferating cells, e.g., HEK293 cells (Extended Data Fig. 9a). Live cell imaging of hiPS-CMs overexpressing 4F over 96 $\mathrm{h}$ showed GFP expression during the $\mathrm{M}$ phase, which reaches the maximum intensity during cytokinesis; in contrast, there is no GFP expression observed in lacZ-treated cells (supplementary movie 1\&2). In line with a previous report ${ }^{20}$, live imaging of hiPS-CMs treated with $4 \mathrm{~F}$ indicated that $70 \%$ of the GFP expressing cells completed cytokinesis while the remaining 30\% were stuck in mitosis without completing cytokinesis (Extended Data Fig. 9b and supplementary movie 1\&2). Fixation and Immunostaining demonstrated that $36 \mathrm{~h}$ post-infection with 4F, the GFP signal is co-localized with the AurKB protein expression at the midbody during mitosis (Extended Data Fig. 9c-d). AurKB protein expression fades after two days post-infection; however, the GFP protein remained and marked that $20-30 \%$ of hiPS-CMs infected with 4F adenovirus, with a decline in the GFP signal after day 4 (Extended Data Fig. 9e-f). EDU nuclei labeling was observed in GFP positive cells (Extended Data Fig. 9e), indicating the S-phase's completion before entering the $\mathrm{M}$ phase.

After demonstrating the AurKB promoter region's ability to reliably indicate mitosis $(100 \%)$ and cytokinesis (70\%) events in cardiomyocytes through transient expression of GFP, we developed a permanent marker for mitosis to be used in vivo and in situ. To this end, we developed a double reporter system to track mitotic events in situ and in vivo based on the AurKB promoter described above. In this 
reporter system, we cloned a Lox-DsRed-Stop-Lox-GFP construct under CAG promoter in lentivirus; in another lentiviral construct, we cloned the Cre encoding protein sequence under the influence of the AurKB promoter (AurKB-Cre) (Extended Data Fig. 10a). Using this double reporter system, all infected cells will become DsRed positive; when mitosis occurs, Cre recombinase will be expressed and will cut out the DsRed-Stop sequence, turning these cardiomyocytes permanently into GFP positive cardiomyocytes. Therefore, the presence of green cells will be an indication of mitotic events. We first validated the color switch in normally proliferating cells (HEK293) (Extended Data Fig. 10b). Then, we further validated the efficiency of this reporter system in detecting mitotic events induced by $4 \mathrm{~F}$ in hiPS-CMs (Extended Data Fig. 10c-d) and pig heart tissue in situ using our recently developed heart slices culture system ${ }^{23,24}$ (Extended Data Fig. 10e-f). This reporter system indicates the number of infected cells (total red-and green-labeled cells) and the number of mitotic events (green-labeled cells). Therefore, the quotient of green and red cardiomyocytes provides a quantification of mitotic cardiomyocytes.

\section{TNNT2-4Fpolyscistronic-NIL induces cardiomyocyte proliferation, improves cardiac function, and reduces scar size in a porcine model of heart failure}

As a proof of concept for our approach's efficacy in inducing transient cardiomyocyte proliferation in large animals, we injected TNNT2-4Fpolyscistronic-NIL or control LacZ-NIL into the pig myocardium one week after induction of myocardial infarction with a 90-min coronary occlusion followed by reperfusion (Fig. 6a). The double reporter system was co-injected into the border zone with the therapeutic or control virus to assess the extent of cardiomyocyte proliferation induced by the TNNT2-4Fpolyscistronic-NIL.

Four weeks post-treatment, every pig treated with TNNT2-4Fpolyscistronic-NIL showed significant improvement in gross heart failure measures such as HW-BW and LW/BW (Fig. 6b). Furthermore, the cardiac functional parameter, ejection fraction, assessed by blinded echocardiography (Fig. 6c) and MRI (Fig. 6d, Extended Data Fig. 11 and supplementary movies 3-6) demonstrated significant improvement in animals treated with TNNT2-4Fpolyscistronic-NIL compared to control virus-treated pigs. Also, TNNT24 Fpolyscistronic-NIL treated pigs exhibited a $25 \%$ reduction in scar size compared to control pigs (Fig. $6 e)$.

TNNT2-4Fpolyscistronic-NIL-treated animals showed that $30 \%$ of the total labeled cardiomyocytes with the double reporter system at the injection site are GFP positive, indicating the cardiomyocyte mitotic activation. In contrast, almost no background proliferation was detected in control virus-treated hearts (Fig. 6f-h), supporting the concept that the improvement in function is due to induction of cardiomyocyte proliferation.

\section{Discussion}

Direct induction of the cell cycle using $4 \mathrm{~F}$ is one of the most robust methods in inducing cardiomyocyte proliferation ${ }^{25,26}$; however, it is essential to understand the mechanism of action of this potential heart failure gene therapy and to tightly control its timing, dosage, and specificity of expression in 
cardiomyocytes. Here, we describe the essential processes associated with forced cardiomyocyte proliferation following cell cycle induction, including sarcomeric disassembly and metabolic reprogramming, in a temporal sequence and on a single cell transcriptomic level. Furthermore, we provide the first proof of concept for this approach's efficacy in improving cardiac function after infarction in a large animal model using a transient and cardiac-specific gene therapy approach.

Understanding the process of human cardiomyocyte proliferation and the reprogramming steps needed for the cardiomyocytes to complete the process is essential to advance the field of cardiac regeneration. Several efforts comparing proliferating fetal cardiomyocytes and adult cardiomyocytes have yielded a certain degree of understanding of the process ${ }^{25}$. However, the highly variable nature of fetal and adult cardiomyocytes limits the ability to elucidate the reprogramming events during proliferation. In the present study, we attempted to identify the essential reprogramming events associated with cell cycle induction by monitoring the same human cardiomyocytes during proliferation on a single-cell transcriptomic level. First, we found that sarcomeric disassembly is an essential step for cardiomyocytes cytokinesis. This finding is consistent with the recent suggestion that proteins responsible for sarcomere assembly, e.g., ephrin-B1, are essential elements for the cell cycle blockade in adult cardiomyocytes as described in a recent preprint ${ }^{27}$.

Furthermore, we demonstrated that proliferating cardiomyocytes shift their metabolism from energy production to biosynthesis. This finding is consistent with the need for new building blocks for cell growth and division. Recent studies suggest that glycolysis ${ }^{28,29}$, glucose oxidation ${ }^{30}$, and the mevalonate pathway ${ }^{31}$ influence myocyte proliferation; our results build upon these previous reports and support the idea that metabolic activity changes may be required for successful myocyte division. Interestingly, a recent paper demonstrated switching the metabolic substrate from fatty acids to glucose induces cardiomyocyte cell-cycle progression ${ }^{30}$. Considering that glucose is a primary biosynthetic substrate in cardiomyocytes $^{32}$, this supports our finding that the switch from catabolic to biosynthetic activities is essential for cell cycle progression in cardiomyocytes.

The advantage of these single-cell RNAseq data is that we reached a time resolution that enabled us to compare two subpopulations of cardiomyocytes, both of which received $4 \mathrm{~F}$ for 48 hours; one subpopulation was delayed entering mitosis while the other subpopulation was in mitosis. This comparison will impact the field of cardiac regeneration and has been long-awaited. This comparison was not possible before because there was no approach to induce cardiomyocyte regeneration that reached the efficiency we achieved with the $4 \mathrm{~F}$. Furthermore, direct induction of cell cycle is a clean approach to understand the mechanism of cardiomyocyte proliferation, unlike other approaches that have many off-target effects, e.g., manipulations of $Y A P^{33}$, a developmental gene that induces dedifferentiation, and use of microRNAs ${ }^{6}$, which have multiple off-target effects.

The insights we gained into the process of cardiomyocyte proliferation and the ability to define the $15 \%$ cardiomyocyte subpopulation that temporarily undergoes mitosis at $48 \mathrm{~h}$ (mitotic subpopulation) and track the reprogramming events in this subpopulation motivated us to perform the next translational 
steps: that is to provide a proof of concept for the efficacy of this approach in improving cardiac function in animal models of heart failure. Interestingly, a recent study showed that AAV-mediated expression of microRNA-199a in infarcted pig hearts initially stimulates cardiac repair through induction of cardiomyocyte proliferation; however, subsequent persistent and uncontrolled expression of the microRNA resulted in sudden arrhythmic death of most of the treated pigs ${ }^{6}$. Our previous in vitro and in vivo results show that myocytes undergo only one round of division after transduction with cell cycle factors because the overexpression of the $4 \mathrm{~F}$ in cardiomyocytes is self-limiting through proteasomemediated degradation of the protein products ${ }^{4}$. Therefore, to perform these translational steps, we needed to transiently and specifically express $4 \mathrm{~F}$ in cardiomyocytes to induce one cycle of proliferation and avoid any adverse effects in other tissues. Over the past decade, there have been significant advances in genetherapy delivery approaches for transient gene expression using either ModRNA ${ }^{34}$ or $\mathrm{NIL}^{13}$. Although the modified RNA approach is a promising virus-free delivery system for transient expression, its poor delivery and specificity to cardiomyocytes limit its applicability. Therefore, NIL was the tool of choice for our animal studies for transient expression with high infection efficiency, as reviewed $\mathrm{in}^{35}$. Our data show that a polycistronic TNNT2-4Fpolycictronic-NIL induces 4F expression only in cardiomyocytes both in vitro and in vivo. Furthermore, TNNT2-4Fpolycistronic-NIL induces proliferation markers in hiPS-CMs, cardiomyocyte cytokinesis in vivo in MADM mice, and significantly improves cardiac function in both a rat and a pig of heart failure caused by myocardial infarction.

In conclusion, we have provided a mechanistic understanding of the process of forced cardiomyocyte proliferation and advanced the clinical feasibility of the $4 \mathrm{~F}$ gene therapy approach for heart failure treatment by minimizing the oncogenic potential of the cell cycle factors using a novel transient and cardiomyocyte-specific viral construct. Further studies are needed to prove effectiveness and safety in more chronic heart failure models in large animals. These studies will pave the way for the first test of this promising approach in patients with ischemic cardiomyopathy.

\section{Limitations of the study}

The use of 60-day mature hiPS-CMs instead of adult primary human cardiomyocytes in the mechanistic studies is a limitation. However, there is a lack of a reliable long-term culture model of adult human cardiomyocytes and the inability to perform single-cell RNAseq on adult cardiomyocytes other than single nuclear RNAseq due to the large size of the adult cardiomyocytes. More importantly, nuclei are at different integrities during the cell cycle stages, which will lead to difficulties in isolating mitotic nuclei and will obfuscate the interpretation of any nuclear RNAseq. Therefore, we used the hiPS-CMs, which are highly pure cardiomyocyte cultures obtained from Cellular Dynamics, Inc. These cells are selected after differentiation using an a-MHC-Blastocidin selection cassette. This strategy yields nearly $100 \%$ pure iPSCCMs, as indicated in our TNNT2 immunostaining images and single-cell RNAseq data. For consistency, only cells that mature for at least 60 days were used for experiments. After this time, the cells have matured to a point at which they have minimal proliferation capacity and minimal basal expression of 
cyclins and $\mathrm{Cdks}^{4}$. Thus, the use of hiPS-CMs provided a homogenous cell population as a starting material to track the reprogramming events during cardiomyocyte proliferation.

The data from the AurKB must be interpreted cautiously as mitotic rather than cytokinesis events. As we described here, there is a $30 \%$ overestimation in cytokinesis events reported by this reporter. Nevertheless, as we show here, the reporter reliably estimates cell cycle induction and mitotic entrance in cardiomyocytes in vitro, in situ, and in vivo in large animal models with almost no background labeling in the control groups. We preferred to use the CAG promoter for this reporter rather than TNNT2 due to the delayed kinetics of the TNNT2 promoter (Extended Data Fig. 5b-c), which could complicate the experimental design and interpretation of results.

All functional efficacy and specificity of expression have been assessed four weeks after injection in an acute heart failure model where the virus was injected one-week post-I/R. Therefore, an assessment of the safety and functional efficacy of the TNNT2-4Fpolycystronic-NIL for a longer time (4-6 months) will be needed. Besides, there is a need for further studies to assess the efficacy of the TNNT2-

4Fpolycystronic-NIL in a more chronic heart failure model where the virus is injected one or two months after I/R.

\section{Methods}

\section{Cloning and preparation of integrating and non-integrating lentivirus.}

cDNA of CAG promoter, TNNT2 promoter, Cre recombinase, AurKB promoter, CCNB, CCND, CDK1, and CDK4 were cloned into the pLenti-MCS-SV-puro backbone (Addgene). To produce the Lentivirus particles, $5 \times 10^{6}$ HEK293 cells were transfected using FuGENE HD transfection reagent (Promega) along with $5 \mu \mathrm{g}$ pMD2.g, 5ug psPAX2 (Integrating lentivirus) or psPAX2-D64V (non-integrating Lentivirus) (Addgene) and $10 \mu \mathrm{g}$ of the expression pLenti vector encoding the gene of interest for $48 \mathrm{~h}$. The media containing the virus were collected and filtered through a Nalgene syringe filter $0.45 \mu \mathrm{m}$. For in vitro experiments, the virus was mixed with polybrene transfecting reagent $(1 \mu \mathrm{g} / \mathrm{ml}$ ) (Millipore Cat\# 1003). The virus was then used to treat 60 days old hiPS-CMs. For in vivo injections, the virus solution was centrifuged at 20,000xg at $4^{\circ} \mathrm{C}$ for $2 \mathrm{~h}$ and the pellet was resuspended in PBS. Modified RNA was synthesized and purchased from Bio-Synthesis, Inc. RNA modifications, transfection reagents, and procedures were conducted following the protocol described in ${ }^{10}$.

\section{Preparation and maintenance of hiPS-CMs}

The human-induced pluripotent stem cell-derived cardiomyocytes (hiPS-CMs) were purchased from Cellular Dynamics (Currently FujiFilm Inc.). These hiPS-CMs have been selected after differentiation using an a-MHC-Blastocidin selection cassette. This strategy yields nearly $100 \%$ pure hiPS-CMs. hiPS-CMs were re-plated on fibronectin-coated plates and cultured in R.P.M.I. medium with a B27 supplement. Then the cardiomyocytes were maintained in RPMI/B27 supplement with insulin for 2-3 months. The cells were transferred every 2-3 weeks on fresh fibronectin. 


\section{Bulk RNAseq analysis}

RNA from hiPS-CMs were isolated using the Qiagen, miRNeasy Micro Kit, \#210874, following homogenization of the tissue in QIAzol (Qiagen). Using the Ovation RNA-seq System v2 Kit (NuGEN), total RNA (20-50 ng) was reverse transcribed to synthesize the first-strand cDNA using a combination of random hexamers and a poly-T chimeric primer. The RNA template was then partially degraded by heating, and the second-strand cDNA was synthesized using DNA polymerase. Double-stranded DNA was then amplified using single primer isothermal amplification (S.P.I.A.). Random hexamers were then used to amplify the second-strand cDNA linearly. cDNA samples were fragmented to an average size of $200 \mathrm{bp}$ using the Covaris $\mathrm{S} 2$ sonicator. Libraries were made from the fragmented cDNA using the Ovation Ultralow V2 kit (NuGen).

Following end-repair and ligation, the libraries were PCR amplified with 9 cycles. A Bioanalyzer assessed library quality on High-Sensitivity DNA chips (Agilent), and concentration was quantified by qPCR (K.A.P.A.). The libraries were sequenced on a Novaseq sequencer with a single-read, 50-cycle sequencing run (Illumina). We utilized the RNAseq-analysis pipeline reported previously ${ }^{15}$. For the readers' convenience and completeness of the current manuscript, we review the pipeline's critical steps/features and statistics below. Available adapters and low-quality regions of reads were trimmed using Fastq-mcf (http://code.google.com/p/ea-utils). Sample QC was assessed using FastQC

(http://www.bioinformatics.babraham.ac.uk/projects/fastqc/). Reads were aligned to the GRCh38 genome using Tophat 2.0.13. Gene expression was tallied by Subread feature Counts using Ensemble's gene annotation for Sscrofa11.1. Finally, we calculated differential expression P-values using edgeR 8. Here, we first filtered out any genes without at least two samples with a C.P.M. (counts per million) between 0.5 and 5000. C.P.M.s below 0.5 indicate nondetectable gene expression, and C.P.M.s above 5000 are typically only seen in mitochondrial genes. If these high-expression genes were not excluded, their counts would disproportionately affect the normalization. After excluding these genes, we renormalized the remaining ones using "calcNormFactor" in edgeR, then calculated P-values for each gene with differential expression between samples using edgeR's assumed negative-binomial distribution of gene expression. We calculated the false discovery rates (FDRs) for each P-value with the Benjamini-Hochberg method based on the built-in R function "p.adjust".

\section{Contractile function monitoring of hiPS-CMs using CardioExcyte 96 system}

hiPS-CMs were plated on fibronectin-coated NSP-96, CardioExcyte 96 Sensor Plates with extra stimulation electrode. The NSP-96 plate was maintained in the CardioExcyte 96 system at $37^{\circ} \mathrm{C}$ and $5 \%$ $\mathrm{CO}_{2}$. The cells were electrically stimulated at $1 \mathrm{~Hz}$ during the experiments. Impedance recordings were performed every 30 minutes for $96 \mathrm{~h}$. The data were analyzed with CardioExcyte 96 analysis software.

\section{Electrophysiological recordings of the ionic current of hiPS-CMs}

Sodium current $\left(\mathrm{I}_{\mathrm{Na}}\right)$ was recorded in the whole-cell configuration of the patch-clamp technique. All recordings were performed at room temperature $\left(20^{\circ}\right.$ to $\left.22^{\circ} \mathrm{C}\right)$. The pipette solution consisted of (in 
$\mathrm{mmol} / \mathrm{L}$ ): $120 \mathrm{KCl}, 1 \mathrm{MgCl}_{2}$, 3MgATP, 10 Hepes, 10 EGTA, pH 7.3. Bath solution contained (in mmol/L) $140 \mathrm{NaCl}, 5.4 \mathrm{KCl}, 1.2 \mathrm{KH}_{2} \mathrm{PO} 4,5 \mathrm{HEPES}, 5.55$ glucose, $1 \mathrm{MgCl}_{2}, 1.8 \mathrm{CaCl}_{2}, \mathrm{pH} 7.4$. $\mathrm{R}_{\text {series }}$ was compensated $>80 \%$; no corrections were made for liquid junction potentials. Data were analyzed with either Clampfit 11 (Molecular Devices) or Igor, dPatch/SutterPatch (Sutter Instruments).

\section{Single-Cell RNA-Sequencing Library Generation}

hiPS-CMs either infected with LacZ or 4F for the indicated time were filtered using a $70 \mu \mathrm{M}$ filter, resuspended in 1\% B.S.A. in PBS, and counted immediately before library preparation. G.E.M. Generation and Barcoding were performed following the Chromium Single Cell" Reagents Kits v3 Rev A User Guide with Chromium Single Cell" G.E.M., Library and Gel Bead Kit v3 (10X Cat \# 1000092). Briefly, the Chromium chip was loaded, and the Chromium Single Cell B program was selected. The chip was ejected immediately following completion of the program. $100 \mu \mathrm{L}$ of recovered G.E.M.s was slowly pipetted and transferred to tubes precooled on ice. The G.E.M.s were then incubated for the R.T. reaction using a thermal cycler at $53^{\circ} \mathrm{C}$ for $45 \mathrm{~min}$, then $85^{\circ} \mathrm{C}$ for $5 \mathrm{~min}$, followed by a $4^{\circ} \mathrm{C}$ hold. Samples were then stored at $-20^{\circ} \mathrm{C}$ overnight. All libraries were pooled and sequenced using the NovaSeq to a read depth of at least 50,000 reads per cell. The sequenced reads for each sample were mapped to the GRCh38 genome to generate the (gene-by-cell) count matrix using cell ranger count (Cell Ranger version 3.1.0 from 10x Genomics) with default parameters. The counts" matrices across the samples were aggregated using cell ranger aggr. The resulting files were processed in $\mathrm{R}$ using the package Seurat (version 3.1.3) ${ }^{36}$. All cells with at least three detected genes and less than $30 \%$ of reads from mitochondrial genes and all detected genes in at least 200 cells were used in the further analyses. The remaining data were normalized using the "LogNormaliz" method. Principal Component Analysis for the subset of the 2000 most variable genes (Seurat function FindVariableFeatures) was then performed on the scaled data. The cells were clustered using the Louvain Algorithm with the resolution parameter value of 0.5 (Seurat function FindClusters) after determining the shared nearest neighbor graph using the first ten principal components (Seurat function FindNeighbors). The data were visualized using the UMAP algorithm with the first ten principal components as input (Seurat function RunUMAP). The cells were grouped into ten clusters based on the distribution of expression of the cell cycle genes of interest. Differential analysis between all pairs of clusters was performed using the Wilcoxon rank-sum test to identify the differentially expressed genes (Seurat function FindMarkers). The dimensionality reduction results were reformatted for compatibility with the learn_graph function in the R package monocle3, used for trajectory analysis ${ }^{37}$. This analysis was done for five groups of cells $-24 \mathrm{~h}$ unique cluster, $48 \mathrm{~h}$ pre-mitotic cells, $48 \mathrm{~h}$ mitotic cells, $72 \mathrm{~h}$ unique cluster, and $48 \mathrm{~h}$ quiescent cells.

\section{Metabolic flux assessment}

The bioenergetics of hiPS-CMs were measured using a Seahorse Bioscience XF96e Flux Analyzer. For these experiments, the assay medium consisted of unbuffered phenol red-free DMEM pH 7.4, supplemented with $5 \mathrm{mM}$ glucose, $1 \mathrm{mM}$ glutamine, and $100 \mu \mathrm{M}$ L-carnitine, $100 \mathrm{nM}$ insulin, and $100 \mu \mathrm{M}$ BSA-palmitate. Following microplate insertion, the XF96e automated protocol consisted of a 12 min delay 
followed by baseline oxygen consumption rate (OCR) and extracellular acidification rate (ECAR) measurements. All experiments were conducted at $37^{\circ} \mathrm{C}$. Data were normalized to the protein content.

\section{Stable isotope-resolved metabolomics (SIRM)}

hiPS-CMs were incubated in growth medium containing $5.5 \mathrm{mM}^{13} \mathrm{C}_{6}$-glucose in 6-well plates for $8 \mathrm{~h}$, after which cell reactions were quenched in cold acetonitrile, and extracted in acetonitrile: water: chloroform ( $\mathrm{v} / \mathrm{v} / \mathrm{v}, 2: 1.5: 1)$, and processed as described previously for metabolite assessments using mass spectropmetry ${ }^{38-42}$. Stable isotope data analyses were performed by obtaining the mass spectrometer .raw files, which are first converted to .mzML format with msConvert tool, a part of an open-source ProteoWizard suite, described in detail by Adusumilli and Mallick ${ }^{43}$. Isotopologue peak deconvolution and assignments were performed using El-MAVEN. Peaks were assigned using a metabolite library first generated and verified using full-scan MS and MS/MS spectra of unlabeled samples, as described previously $41,42,44$. The library contained metabolite names and corresponding molecular formulae used to generate theoretical $\mathrm{m} / \mathrm{z}$ values for all possible isotopologues and retention times. The El-MAVEN parameters for compound library matching were as follows: EIC Extraction Window $\pm 7 \mathrm{ppm}$; Match Retention Time $\pm 0.60 \mathrm{~min}$. For ${ }^{13} \mathrm{C}$ isotopologue peak detection, the software criteria were set as follows: Minimum Isotope-parent correlation 0.5; Isotope is within seven scans of the parent; Abundance threshold 1.0; Maximum Error To Natural Abundance $100 \%$. All assignments were visually inspected and compared with unlabeled samples for reference. Any peak groups assigned in error, e.g., not present or having different retention times than in the unlabeled samples, were deleted, and correct peak assignments were added manually, as described in ${ }^{42}$. Finally, the peak list with corresponding abundances was exported to a comma-separated (CSV) file and uploaded to the Polly workflow to perform natural abundance correction using Polly Isocorrect. Finally, the data were analyzed and plotted with GraphPad Prism 8.0 (GraphPad Software, San Diego, Ca, U.S.A.).

\section{Immunocytochemistry and immunohistochemistry}

The hiPS-CMs were fixed in 4\% formaldehyde for 20 min (Thermos Scientific Cat\#28908). Fixed cells were washed three times with PBS. For animal experiments, the heart was isolated and perfused with $1 \mathrm{M}$ $\mathrm{KCL}$ to arrest the heart, then washed with PBS and perfused with $4 \%$ paraformaldehyde (Electron microscopy sciences Cat\# 15713-S). The hearts were cut longitudinally into half and kept in $4 \%$ paraformaldehyde for $48 \mathrm{~h}$. The hearts were then washed with PBS, then placed in $10 \%$ sucrose solution for $1 \mathrm{~h}$ followed by $20 \%$ sucrose solution for $1 \mathrm{~h}$ at room temperature, then placed in $30 \%$ sucrose solution overnight at $4^{\circ} \mathrm{C}$. The hearts were then processed into frozen OCT blocks and kept at $-80^{\circ} \mathrm{C}$ for $24 \mathrm{~h}$. The heart was sectioned using a cryostat (Leica Inc.) in $8 \mu \mathrm{m}$ thick sections, placed on slides, and kept at $-20^{\circ} \mathrm{C}$ until staining. To start staining, the OCT was removed from the section by heating at $95^{\circ} \mathrm{C}$ for 5 min then washing in PBS for 30 min.

The fixed cells or cleaned sections were permeabilized with $0.1 \%$ Triton X-100 for 15 min (Millipore Cat\# 55163804 ) and then blocked with 3\% bovine serum albumin (B.S.A.) in PBS for 60 min at room 
temperature (V.W.R. Cat\# 0332). The cells or tissue sections were then probed with primary antibody (1:200 in 1\% B.S.A.) for 1.5 h. Then washed three times with PBS. They were then labeled with secondary fluorescent antibody (1:200 in 1\% B.S.A.). Table 1 showed a list of primary and secondary antibodies used in this study. Cells/ tissue sections were then washed three times with PBS and stained with DAPI $1 \mu \mathrm{g} / \mathrm{ml}$ (Biotium Cat\# 40043) to stain the nucleolus blue. For EDU detection, the cells were also treated with $5 \mu \mathrm{M}$ 5-ethyl-2-deoxyuridine (EDU) for the course of the experiment, which will incorporate into the newly synthesized DNA. After fixation, permeabilization, and blocking of the cells/ tissue sections, the EDU incorporation was visualized using Click it EDU-Alexa-Flour ${ }^{647}$ imaging kit (Thermo Fisher Cat\# C10340). For live-cell imaging, the cells were treated with NucBlue live cells stain (Thermo Fisher) for 20 minutes.

Imaging was conducted for the whole well using the high content imaging instrument, Cytation 1. The percentage of co-localization of PHH3, EDU, GFP, or gene expression and Troponin-T was quantified using Gen 5.05 software.

\begin{tabular}{|l|l|l|}
\hline Specific protein & Primary antibody & Cat \# \\
\hline Cardiac Troponin & Mouse Monoclonal Cardiac Troponin T Antibody & $\begin{array}{l}\text { Thermos Fisher } \\
\text { (MA5-12960) }\end{array}$ \\
\hline Cardiac troponin & $\begin{array}{l}\text { Rabbit monoclonal Anti-Cardiac Troponin T } \\
\text { antibody }\end{array}$ & Abcam ab209813 \\
\hline $\begin{array}{l}\text { Phospho Histon } \\
\text { H3 (PHH) }\end{array}$ & $\begin{array}{l}\text { Rabbit monoclonal Anti-Histone H3 (phospho S10) } \\
\text { antibody - ChIP Grade }\end{array}$ & Abcam ab5176 \\
\hline CDK4 & Rabbit monoclonal Anti-CDK4 & Abcam ab199728 \\
\hline CCNB & Rabbit monoclonal Anti-CCNB & Abcam ab32053 \\
\hline CCND & Rabbit monoclonal Anti-CCND & Abcam ab134175 \\
\hline CDK1 & Mouse monoclonal Anti-CDK1 & Abcam ab18 \\
\hline Arura Kinase B & Rabbit polyclonal anti-Aurora B & Abcam ab2254 \\
\hline Ds Red & Mouse monoclonal anti R.F.P. & Abcam ab150115 \\
\hline
\end{tabular}




\begin{tabular}{|l|l|}
\hline Secondary antibody & Cat \# \\
\hline Goat anti-Mouse IgG (H+L), F.I.T.C. & Thermos fisher A16079 \\
\hline Texas Red Goat anti-Rabbit IgG (H+L) & Thermos fisher T-6391 \\
\hline Alexa Flour ${ }^{\mathrm{TM}} 647$ donkey anti-rabbit IgG & Thermos fisher A31573 \\
\hline Texas Red -X goat anti-mouse IgG & Thermos fisher T862 \\
\hline Anti-GFP, rabbit polyclonal antibody Alexa Fluor ${ }^{\mathrm{TM}} 448$ conjugate & Thermos fisher A21311 \\
\hline
\end{tabular}

\section{qRT-PCR}

A piece of the left ventricle at the injection area was collected for RNA extraction. The heart was homogenized with QIAzol lysis reagent ( Qiagen Cat\# 79306), then RNA was extracted following the miRNeasy micro kit protocol (Qiagen Cat\# 217084). The concentration of the RNA was calculated using the Cytation 1 reader. 1 ug of each RNA sample was used for reverse transcription using a mixture of oligo(dT) and random hexamer primers (SuperScript IV VILO Master Mix, ThermoFisher Scientific Cat \# 11756050). Real-time PCR analysis was conducted with Taqman fast advanced master mix (Thermo Fisher 4444557), and primers specific to human CDK1, CDK4, CCNB, CCND genes (Hs00938777, Hs00364847, Hs01030099, Hs00765553 respectively, Applied Biosystem ), and the expression was normalized to rat GAPDH expression (Rn01775763 Applied Biosystem) using the Quant studio 5 real-time PCR detection system (Applied Biosystems).

\section{Animal experiments}

Animal studies were performed following the University of Louisville animal use guidelines, and the protocols were approved by the Institutional Animal Care and Use Committee (IACUC) and were accredited by the Association for Assessment and Accreditation of Laboratory Animal Care.

\section{MADM mice experiment}

For lineage tracing, we used mosaic analysis with double markers (MADM) transgenic mice were developed as prescribed in ${ }^{4}$. All the surgeries were performed as described in ${ }^{45}, 46$. Adult (about 12 weeks old) female MADM mice were anesthetized with sodium pentobarbital (60 mg/kg i.p.). After opening the chest through a left thoracotomy, a nontraumatic balloon occluder was implanted around the mid-left anterior descending coronary artery (L.A.D.) using an 8-0 nylon suture. Myocardial infarction was produced by 60 -min coronary ischemia, followed by reperfusion (I/R). Rectal temperature was carefully monitored and maintained around $37^{\circ} \mathrm{C}$ throughout the experiment. Successful performance of coronary occlusion and reperfusion was verified by visual inspection and by observing S-T segment elevation and widening of the QRS on the electrocardiogram during ischemia and their resolution after reperfusion. Seven days after I/R, mice were re-anesthetized with sodium pentobarbital, $60 \mathrm{mg} / \mathrm{kg}$ I.P. and the chest 
reopened through a central thoracotomy. The mice were randomly selected to be injected with $20 \mathrm{ul}$ of TNNT2-4Fpolycistronic-NIL, TNNT2-LacZ-NIL virus intramyocardially using a 30-gauge needle. The injections were made at the border between infarcted and non-infarcted myocardium as two injections 10 $\mu \mathrm{L}$ each ( $2 \times 10^{7}$ transducing units (T.U.) per mouse heart). Forty-eight hours after injection, mice received Tamoxifen (40mg/kg I.P.) for three days (Sigma Aldrich T5648). Mice were euthanized 14 days after MI, and the hearts were harvested for pathology study. The mice sergeant was blinded to all administered viruses.

MADM mice frozen hearts were sectioned Longitudinally into 180-210 sections (3 sections per slide collect one and throw away 2) $8 \mu \mathrm{m}$ thick. The frozen sections were fixed, permeabilized, and blocked as described in Immunocytochemistry and immunohistochemistry section, then stained with Dapi $1 \mu \mathrm{g} / \mathrm{ml}$ (Biotium Cat\# 40043) to stain the nucleolus blue. The Coverslips were mounted with Vectashield antifading medium (vector labs Cat\# H-1000) onto slides and visualized using Keyence BZ9000 imaging system (10X magnification to the whole left ventricle). The percentage of single-colored cardiomyocytes from the total labeled cardiomyocytes was calculated using B.Z. analyzer software. Individuals analyzed the results were blinded to the treatment applied in each animal.

\section{Rat experiments}

All surgeries were performed as described in ${ }^{47,48}$. Briefly, Female Fischer 344 (F344) rats at the age ranging from 8-12 weeks were anesthetized with ketamine $(37 \mathrm{mg} / \mathrm{kg})$ and xylazine $(5 \mathrm{mg} / \mathrm{kg})$, intubated, and ventilated with a rodent respirator. Anesthesia was maintained with $1 \%$ isoflurane inhalation, and body temperature was kept at $37^{\circ} \mathrm{C}$ with a heating pad. All rats underwent a $2 \mathrm{~h}$ occlusion of the left anterior descending coronary artery, followed by reperfusion. Seven days after Ml, echocardiography was performed to ensure the development of Ml. All rats in this study had EF drop $>20$ points from baseline. Rats were randomized into two groups (TNNT2-GFP-NIL, TNNT2-4Fpolycistronic-NIL). Rats were reanesthetized with ketamine/xylazine, intubated, and ventilated. The chest was reopened to expose the heart. Viral vectors $\left(1 \times 10^{8}\right.$ T.U. per rat heart in $100 \mu \mathrm{l}$ PBS) were injected into the left ventricle along the infarct border at five sites $(20 \mu \mathrm{l} / \mathrm{site})$ using a $30 \mathrm{G}$ needle. The rat surgeon was blinded to whether $4 \mathrm{~F}$ or control non-integrated lentivirus was administered in each animal.

Cardiac function was assessed by serial echocardiography at baseline (before MI), one week after MI (before virus injection), and then four weeks after virus injection. Animals were anesthetized lightly with isoflurane, placed on the imaging table in the supine position, and prepared for imaging using the Vevo 2100 Imaging System (Visual Sonics) equipped with a 25-MHz transducer. Parasternal longitudinal axis images were acquired and analyzed by LV trace using the Vevo LAB 3.2.6 to obtain the LV functional parameters, including the end-diastolic and end-systolic area, volume, stroke volume, fractional shortening, and ejection fraction. Imaging and calculations were done by an individual who was blinded to the treatment, and the code was broken after all data acquired. 
At the end of the experiments ( 5 weeks after $\mathrm{Ml}$ ), animals were sacrificed, and their hearts were harvested for histological studies. The frozen hearts were sectioned longitudinally into 400-500 sections 8um thickness (take a section to throw one add two sections per slide), and one slide for every ten slides (2025 slide per animal) were stained with Standard Masson's Trichrome staining to determine scar size. The stained sections were imaged using the Keyence BZ9000 imaging system (4X magnification). Image J software was used to measure the scar area (blue) and healthy area (red) on longitudinal sections. Individuals assessing scar area were blinded to the treatment applied in each animal.

\section{Pig experiments}

All surgeries were performed as described in ${ }^{49}$. Yorkshire pigs weighing $25-35 \mathrm{~kg}$ received $200 \mathrm{mg}$ amiodarone orally daily for seven days pre-operatively. Pigs were premedicated with an intramuscular injection of a solution containing ketamine hydrochloride and xylazine.

Pigs were injected with a dose of buprenorphine S.R. before the procedure. To create myocardial infarction, the right neck's skin was cut to make a small opening, allowing access to the right carotid artery. A 7-8F fast-cath sheath was introduced into the carotid artery. The pig was injected with Heparin (300 units $/ \mathrm{kg}$ I.V.) to prevent clotting of the sheaths and catheters during the procedure. After intubation, the pig was mechanically ventilated. Anesthesia was maintained with isoflurane. Body temperature was monitored continuously with a rectal probe attached to a thermocouple and maintained within physiology range using a veterinary blanket. The pigs were subjected to 5 minutes of stabilization, followed by baseline hemodynamics and echocardiography.

A 6-7F Hockey-stick catheter was guided to the left main coronary artery under fluoroscopy as following: the catheter engaged the left main coronary ostium, and an angioplasty-type balloon catheter and guidewire assembly were fluoroscopically guided into the L.A.D. Then, the wire was advanced into the distal L.A.D., and an appropriate balloon catheter was telescoped over the wire and positioned above the first diagonal branch (the entire L.A.D. territory was included for occlusion). The balloon's placement will be verified by intracoronary contrast dye injection (Contrast media) and documented by cine angiogram before inflation. The balloon was inflated to occlude the L.A.D. and the L.A.D. occlusion was maintained for 90 minutes to produce myocardial infarction, targeting an infarct size of $>50 \%$ of the area at risk. Inflation and position of the balloon were verified by contrast angiogram again at the end of ischemia. If necessary, the balloon will be repositioned, and such "positional re-inflation" will be limited to less than 20 seconds to avoid any preconditioning. Once the balloon is inflated, external defibrillator pads were placed on the pig's chest for "hands-free" cardioversion if ventricular fibrillation occurs using a bipolar defibrillator (HP Codemaster XL+) at 300 Joules. After the 90 min ischemic period, the intracoronary balloon was deflated to initiate reperfusion. After the procedure of myocardial infarction, the balloon catheter was withdrawn, and a cine angiogram was taken to document the wide open of the L.A.D. artery. After withdrawing the Hockey-stick guide catheter, the arterial sheath catheter was removed, and the arterial was repaired by anastomosis. The skin incision was closed in 3 layers using 3-0 Vicryl for internal sutures and 3-0 P.D.S. for the final subcutaneous layer. The pig was weaned from anesthesia, and the 
animal was extubated when appropriate and allowed to recover. Animals received antimicrobial therapy cetiofur pre-operatively and every 24 hours for the first 48 hours postoperatively. Animals were prepped and draped in a routine sterile fashion. $5 \%$ dextrose and normal saline was continuously infused during the procedures

Seven days after the MI procedure, pigs were re-anesthetized as described above. Pigs were subjected to MRI scans and echocardiography. Anesthesia was maintained with isoflurane. Body temperature was monitored continuously with a rectal probe attached to a thermocouple and maintained within physiology range using a veterinary blanket. Animals will be prepped and draped in a routine sterile fashion. A dose of buprenorphine S.R. will be given before the procedure. The chest was opened through the initial skin incision and continued down to the sternum. To avoid a post-surgical pulmonary complication, a midline sternotomy to expose the heart in the intrapleural space without breaking the pleural membrane was performed with extra care to avoid rapture of the pleural membrane intact during the opening of the mediastinum. Then the heart suspended in a pericardial cradle as described in ${ }^{50,51}$.

In the case of the pleural membrane broken, efforts are made to close the tear by re-approximation of the pleural membrane with 6-0 Prolene and to reestablish a negative pressure in the pleural cavity using a withdrawal tube with a purse-string closure. After the chest was opened, the pericardium was cut vertically, and the heart was exposed and suspended in a pericardial cradle. Five intramyocardial injections ( $200 \mathrm{ml}$ each) $\left(3 \times 10^{9} \mathrm{~T}\right.$.U. total/pig heart) of viral vectors were performed along the infarct border, and the site of injection was demarcated with a 6-0 Prolene suture. After completing these procedures, warm normal saline (approx. $500 \mathrm{~mL}$ ) was used for flushing the thoracic cavity. This flush was suctioned out before closing the chest. The pericardium was approximated as soon as possible. The sternum was closed with a $20 \mathrm{G}$ stainless steel suture and 5 Green Braided P.T.F.E. nonabsorbable surgical sutures. The chest was closed in layers (0 PDS II suture for the muscle and 2-0 PDS II suture subcutaneously), and a single mediastinal tube ( $18 \mathrm{~F}$ catheter), 3-way valve, and $60 \mathrm{cc}$ syringe will be used to reestablish a negative intrapleural pressure and evacuate any remaining blood or irrigation solution. The chest tube ( $18 \mathrm{~F}$ catheter) was removed before skin closing after no visible air leak or blood accumulation, and a purse-string suture (2-0 PDS II) was used to ensure an airtight seal. The skin incision was then glued with Vetbond adhesive. Then the chest was closed, and the inhaled anesthetic was turned off, the animal extubated when appropriate, and allowed to recover. Animals received antimicrobial therapy cetiofur pre-operatively and every $24 \mathrm{~h}$ for the first $48 \mathrm{~h}$ postoperatively.

Four weeks after virus injection animal was anesthetized with isoflurane as described before to perform the final cardiac MRI and echocardiography. Body temperature will be monitored and maintained within the physiological range. Arterial blood pressure and surface E.C.G. were monitored continuously. Then the animal was deeply anesthetized with $5 \%$ isoflurane. A bolus of $3-6 \mathrm{ml} / \mathrm{kg}$ of $3 \mathrm{M} \mathrm{KCl}$ solution will be injected into the left atrium until the heart is arrested. After the cessation of vital signs, the heart will be harvested for postmortem procession.

\section{T.T.C. stain of the pig heart}


Directly after the heart was harvested, the aorta was perfused with normal saline (500-1000 ml) to flush out vascular blood. The heart was weighed and transversely sliced into 5-6 sections. Heart sections were incubated in $1 \%$ T.T.C. at $37^{\circ} \mathrm{C}$ for $5 \mathrm{~min}$. Then right ventricular, atriums were removed, and LV sections were weighted. The pictures of LV slices were taken using a professional camera. Images were analyzed using Image-J software. Scar size percentages were calculated.

\section{Statistical Analyses}

For all assays, power analyses were performed to choose the group sizes, which will provide $>80 \%$ power to detect a $10 \%$ absolute change in the parameter with a 5\% Type I error rate. These power analyses indicated a minimum of 4 experimental replicates per group; therefore, we used a range of $4-15$ experimental replicates per group for each assay. Special statistical consideration for RNAseq was detailed under the methods section. The Kolmogorov-Smirnov (K-S) test for normality was conducted; all data sets showed normal distribution. Then, differences between the two groups were examined for statistical significance with unpaired Student t-tests. However, to compare data consisting of more than two groups, we performed one- or Two-way ANOVA tests followed by Bonferroni post hoc multiple comparisons to get the corrected $p$-value. A value of $P<0.05$ was regarded as significant. Error bars indicate S.D. The person who performed the analysis was blinded to the experimental groups. Two blinded clinical cardiology fellows assessed all cardiac function Echocardiography and MRI analyses, and the data represented are the average of their analyses. Usually, there were no significant discrepancies between the two readings.

\section{Declarations}

\section{Data availability.}

All sequencing data are deposited in GEO. Bulk RNAseq is under accession number: GSE162165 (reviewer access token (gtafwsayjrgbpet)). Single-cell RNAseq is under accession number: GSE162326 (reviewer access token (wnyrgaesbbadrix)).

\section{Acknowledgments}

T.M.A.M. is supported by NIH grants R01HL147921 and P30GM127607 and American Heart Association grant 16SDG29950012. The authors also acknowledge NIH grants F32HL149140 (R.R.E.A.), P30GM127607 (B.G.H.), R01HL130174 (B.G.H.), R01HL147844 (B.G.H.), R01ES028268 (B.G.H.), P01HL78825 (R.B., B.G.H.), UM1HL113530 (R.B.), and 2U54HL120163. We also acknowledge the U.S.A. Department of Defense for the grant W81XWH-20-1-0419 (J.S., T.M.A.M.). We acknowledge the Genomics core at the University of Louisville for providing access to the 10X Genomics and the Bioinformatics core at Gladstone Institute for performing all the transcriptomics analyses. We would also like to thank the radiology department at the University of Louisville for performing the magnetic resonance imaging.

\section{Author contributions}


R.R.E.A.: collection and analysis of molecular and cellular data, manuscript writing, and final approval of the manuscript; Q.O.: heart cutting, staining, and imaging; X-L.T.: Pig and rat surgery, viral injection, echocardiography; M.S. and K.M.K.: echocardiography and MRI analyses; Y.G., Y.H., and Y.N.: Mouse surgery, modRNA, and viral injection. L.M., P.K.L., and B.G.H.: Metabolic analysis; K.C., and R.T.:

Bioinformatics analyses; B.M.A. and J.S.: Electrophysiology analysis; H.R.J., A.S., Z.I., A.M.S., and S.H.: histology and analyses including staining, imaging, and quantifications. A.S.E.: MRI imaging quantification; K.N.I and D.S.: conception, design, and provided funding of the modRNA experiments; R.B.: Design and supervision of rat and pig experiments; T.M.A.M.: conception and design of the overall work, provided funding. All authors have contributed to the manuscript writing and final approval of the manuscript.

\section{Competing Interests}

T.M.A.M., K.I., and D.S. hold equities in Tenaya Therapeutics.

\section{References}

1. Hydbring P, Malumbres $M$ and Sicinski P. Non-canonical functions of cell cycle cyclins and cyclindependent kinases. Nat Rev Mol Cell Biol. 2016;17:280-92.

2. Malumbres M and Barbacid M. Cell cycle, CDKs and cancer: a changing paradigm. Nat Rev Cancer. 2009;9:153-66.

3. Li VC and Kirschner MW. Molecular ties between the cell cycle and differentiation in embryonic stem cells. Proceedings of the National Academy of Sciences of the United States of America. 2014;111:9503-8.

4. Mohamed TMA, Ang YS, Radzinsky E, Zhou P, Huang Y, Elfenbein A, Foley A, Magnitsky S and Srivastava D. Regulation of Cell Cycle to Stimulate Adult Cardiomyocyte Proliferation and Cardiac Regeneration. Cell. 2018;173:104-116 e12.

5. Zong H, Espinosa JS, Su HH, Muzumdar MD and Luo L. Mosaic analysis with double markers in mice. Cell. 2005;121:479-92.

6. Gabisonia K, Prosdocimo G, Aquaro GD, Carlucci L, Zentilin L, Secco I, Ali H, Braga L, Gorgodze N, Bernini F, Burchielli S, Collesi C, Zandona L, Sinagra G, Piacenti M, Zacchigna S, Bussani R, Recchia FA and Giacca M. MicroRNA therapy stimulates uncontrolled cardiac repair after myocardial infarction in pigs. Nature. 2019;569:418-422.

7. Chien KR, Frisen J, Fritsche-Danielson R, Melton DA, Murry CE, and Weissman IL. Regenerating the field of cardiovascular cell therapy. Nature biotechnology. 2019;37:232-237.

8. Eschenhagen T, Bolli R, Braun T, Field LJ, Fleischmann BK, Frisen J, Giacca M, Hare JM, Houser S, Lee RT, Marban E, Martin JF, Molkentin JD, Murry CE, Riley PR, Ruiz-Lozano P, Sadek HA, Sussman MA and Hill JA. Cardiomyocyte Regeneration: A Consensus Statement. Circulation. 2017;136:680686. 
9. Sada H, Ban T, Fujita T, Ebina Y, and Sperelakis N. Developmental change in fast Na channel properties in embryonic chick ventricular heart cells. Can J Physiol Pharmacol. 1995;73:1475-84.

10. Sultana N, Magadum A, Hadas Y, Kondrat J, Singh N, Youssef E, Calderon D, Chepurko E, Dubois N, Hajjar RJ and Zangi L. Optimizing Cardiac Delivery of Modified mRNA. Mol Ther. 2017;25:13061315.

11. Xu Z, Chen F, Zhang L, Lu J, Xu P, Liu G, Xie X, Mu W, Wang Y and Liu D. Non-integrating lentiviral vectors based on the minimal S/MAR sequence retain transgene expression in dividing cells. Sci China Life Sci. 2016;59:1024-1033.

12. Nightingale SJ, Hollis RP, Pepper KA, Petersen D, Yu XJ, Yang C, Bahner I, and Kohn DB. Transient gene expression by nonintegrating lentiviral vectors. Mol Ther. 2006;13:1121-32.

13. Shaw A and Cornetta K. Design and Potential of Non-Integrating Lentiviral Vectors. Biomedicines. 2014;2:14-35.

14. Milone MC and O'Doherty U. Clinical use of lentiviral vectors. Leukemia. 2018;32:1529-1541.

15. Mohamed TM, Stone NR, Berry EC, Radzinsky E, Huang Y, Pratt K, Ang YS, Yu P, Wang H, Tang S, Magnitsky S, Ding S, Ivey KN and Srivastava D. Chemical Enhancement of In Vitro and In Vivo Direct Cardiac Reprogramming. Circulation. 2017;135:978-995.

16. Ali SR, Hippenmeyer S, Saadat LV, Luo L, Weissman IL, and Ardehali R. Existing cardiomyocytes generate cardiomyocytes at a low rate after birth in mice. Proc Natl Acad Sci U S A. 2014;111:8850-5.

17. Nguyen NUN, Canseco DC, Xiao F, Nakada Y, Li S, Lam NT, Muralidhar SA, Savla JJ, Hill JA, Le V, Zidan KA, El-Feky HW, Wang Z, Ahmed MS, Hubbi ME, Menendez-Montes I, Moon J, Ali SR, Le V, Villalobos E, Mohamed MS, Elhelaly WM, Thet S, Anene-Nzelu CG, Tan WLW, Foo RS, Meng X, Kanchwala M, Xing C, Roy J, Cyert MS, Rothermel BA, and Sadek HA. A calcineurin-Hoxb13 axis regulates growth mode of mammalian cardiomyocytes. Nature. 2020.

18. Ma HT and Poon RY. How protein kinases co-ordinate mitosis in animal cells. Biochem J. 2011;435:17-31.

19. Canseco DC, Kimura W, Garg S, Mukherjee S, Bhattacharya S, Abdisalaam S, Das S, Asaithamby A, Mammen PP, and Sadek HA. Human ventricular unloading induces cardiomyocyte proliferation. $J$ Am Coll Cardiol. 2015;65:892-900.

20. Hesse M, Doengi M, Becker A, Kimura K, Voeltz N, Stein V, and Fleischmann BK. Midbody Positioning and Distance Between Daughter Nuclei Enable Unequivocal Identification of Cardiomyocyte Cell Division in Mice. Circulation Research. 2018;123:1039-1052.

21. Jiang J, Burgon PG, Wakimoto H, Onoue K, Gorham JM, O'Meara CC, Fomovsky G, McConnell BK, Lee RT, Seidman JG, and Seidman CE. Cardiac myosin binding protein $\mathrm{C}$ regulates postnatal myocyte cytokinesis. Proceedings of the National Academy of Sciences of the United States of America. 2015;112:9046-51.

22. Kimura M, Uchida C, Takano Y, Kitagawa M, and Okano Y. Cell cycle-dependent regulation of the human aurora B promoter. Biochem Biophys Res Commun. 2004;316:930-6. 
23. Ou Q, Abouleisa RRE, Tang XL, Juhardeen HR, Meki MH, Miller JM, Giridharan G, El-Baz A, Bolli R, and Mohamed TMA. Slicing and Culturing Pig Hearts under Physiological Conditions. J Vis Exp. 2020.

24. Ou Q, Jacobson Z, Abouleisa RRE, Tang XL, Hindi SM, Kumar A, Ivey KN, Giridharan G, El-Baz A, Brittian K, Rood B, Lin YH, Watson SA, Perbellini F, McKinsey TA, Hill BG, Jones SP, Terracciano CM, Bolli R and Mohamed TMA. Physiological Biomimetic Culture System for Pig and Human Heart Slices. Circulation Research. 2019;125:628-642.

25. Sadek H and Olson EN. Toward the Goal of Human Heart Regeneration. Cell Stem Cell. 2020;26:7-16.

26. Hsieh PCH and Kamp TJ. To Be Young at Heart. Cell Stem Cell. 2018;22:475-476.

27. Cauquil M, Mias C, Guilbeau-Frugier C, Karsenty C, Seguelas M-H, Genet G, Renaud-Gabardos E, Prats A-C, Pons V, Branchereau M, Heymes C, Calise D, Lairez O, Daviaud D, Honton B, Frongia C, Ducommun B, Delisle M-B, Arvanitis DN, Pathak A, Sénard J-M and Galés C. Ephrin-B1 blocks adult cardiomyocyte proliferation and heart regeneration. bioRxiv. 2019:735571.

28. Magadum A, Singh N, Kurian AA, Munir I, Mehmood T, Brown K, Sharkar MTK, Chepurko E, Sassi Y, Oh JG, Lee P, Santos CXC, Gaziel-Sovran A, Zhang G, Cai CL, Kho C, Mayr M, Shah AM, Hajjar RJ, and Zangi L. Pkm2 regulates cardiomyocyte cell cycle and promotes cardiac regeneration. Circulation. 2020; In press.

29. Honkoop H, de Bakker DE, Aharonov A, Kruse F, Shakked A, Nguyen PD, de Heus C, Garric L, Muraro MJ, Shoffner A, Tessadori F, Peterson JC, Noort W, Bertozzi A, Weidinger G, Posthuma G, Grun D, van der Laarse WJ, Klumperman J, Jaspers RT, Poss KD, van Oudenaarden A, Tzahor E, and Bakkers J. Single-cell analysis uncovers that metabolic reprogramming by ErbB2 signaling is essential for cardiomyocyte proliferation in the regenerating heart. Elife. 2019;8.

30. Cardoso AC, Lam NT, Savla JJ, Nakada Y, Pereira AHM, Elnwasany A, Menendez-Montes I, Ensley EL, Petric UB, Sharma G, Sherry AD, Malloy CR, Khemtong C, Kinter MT, Tan WLW, Anene-Nzelu CG, Foo RSY, Nguyen NUN, Li S, Ahmed MS, Elhelaly WM, Abdisalaam S, Asaithamby A, Xing C, Kanchwala M, Vale G, Eckert KM, Mitsche MA, McDonald JG, Hill JA, Huang L, Shaul PW, Szweda LI, and Sadek HA. Mitochondrial substrate utilization regulates cardiomyocyte cell-cycle progression. Nature Metabolism. 2020;2:167-178.

31. Mills RJ, Parker BL, Quaife-Ryan GA, Voges HK, Needham EJ, Bornot A, Ding M, Andersson H, Polla M, Elliott DA, Drowley L, Clausen M, Plowright AT, Barrett IP, Wang QD, James DE, Porrello ER, and Hudson JE. Drug Screening in Human PSC-Cardiac Organoids Identifies Pro-proliferative Compounds Acting via the Mevalonate Pathway. Cell stem cell. 2019;24:895-907 e6.

32. Tran DH and Wang ZV. Glucose Metabolism in Cardiac Hypertrophy and Heart Failure. J Am Heart Assoc. 2019;8:e012673.

33. Monroe TO, Hill MC, Morikawa Y, Leach JP, Heallen T, Cao S, Krijger PHL, de Laat W, Wehrens XHT, Rodney GG, and Martin JF. YAP Partially Reprograms Chromatin Accessibility to Directly Induce Adult Cardiogenesis In Vivo. Dev Cell. 2019;48:765-779 e7.

34. Zangi L, Lui KO, von Gise A, Ma Q, Ebina W, Ptaszek LM, Später D, Xu H, Tabebordbar M, Gorbatov R, Sena B, Nahrendorf M, Briscoe DM, Li RA, Wagers AJ, Rossi DJ, Pu WT and Chien KR. Modified 
mRNA directs the fate of heart progenitor cells and induces vascular regeneration after myocardial infarction. Nat Biotechnol. 2013;31:898-907.

35. Milone MC and O'Doherty U. Clinical use of lentiviral vectors. Leukemia. 2018.

36. Stuart T, Butler A, Hoffman P, Hafemeister C, Papalexi E, Mauck WM, 3rd, Hao Y, Stoeckius M, Smibert P, and Satija R. Comprehensive Integration of Single-Cell Data. Cell. 2019;177:1888-1902 e21.

37. Cao J, Spielmann M, Qiu X, Huang X, Ibrahim DM, Hill AJ, Zhang F, Mundlos S, Christiansen L, Steemers FJ, Trapnell C, and Shendure J. The single-cell transcriptional landscape of mammalian organogenesis. Nature. 2019;566:496-502.

38. Lane AN, Fan TW, Xie Z, Moseley HN, and Higashi RM. Isotopomer analysis of lipid biosynthesis by high resolution mass spectrometry and NMR. Analytica chimica acta. 2009;651:201-8.

39. Le A, Lane AN, Hamaker M, Bose S, Gouw A, Barbi J, Tsukamoto T, Rojas CJ, Slusher BS, Zhang H, Zimmerman LJ, Liebler DC, Slebos RJ, Lorkiewicz PK, Higashi RM, Fan TW, and Dang CV. Glucoseindependent glutamine metabolism via TCA cycling for proliferation and survival in B cells. Cell Metab. 2012;15:110-21.

40. Lorkiewicz P, Higashi RM, Lane AN, and Fan TW. High information throughput analysis of nucleotides and their isotopically enriched isotopologues by direct-infusion FTICR-MS. Metabolomics. 2012;8:930-939.

41. Salabei JK, Lorkiewicz PK, Mehra P, Gibb AA, Haberzettl P, Hong KU, Wei X, Zhang X, Li Q, Wysoczynski M, Bolli R, Bhatnagar A, and Hill BG. Type 2 Diabetes Dysregulates Glucose Metabolism in Cardiac Progenitor Cells. J Biol Chem. 2016;291:13634-48.

42. Lorkiewicz PK, Gibb AA, Rood BR, He L, Zheng Y, Clem BF, Zhang X, and Hill BG. Integration of flux measurements and pharmacological controls to optimize stable isotope-resolved metabolomics workflows and interpretation. Scientific Reports. 2019;9:13705.

43. Adusumilli R and Mallick P. Data Conversion with ProteoWizard msConvert. Methods Mol Biol. 2017;1550:339-368.

44. Wei X, Lorkiewicz PK, Shi B, Salabei JK, Hill BG, Kim S, McClain CJ and Zhang X. Analysis of stable isotope assisted metabolomics data acquired by high resolution mass spectrometry. Analytical Methods. 2017;9:2275-2283.

45. Mehra P, Guo Y, Nong Y, Lorkiewicz P, Nasr M, Li Q, Muthusamy S, Bradley JA, Bhatnagar A, Wysoczynski M, Bolli R, and Hill BG. Cardiac mesenchymal cells from diabetic mice are ineffective for cell therapy-mediated myocardial repair. Basic Res Cardiol. 2018;113:46.

46. Li Q, Guo Y, Ou Q, Wu WJ, Chen N, Zhu X, Tan W, Yuan F, Dawn B, Luo L, Hunt GN and Bolli R. Gene transfer as a strategy to achieve permanent cardioprotection II: rAAV-mediated gene therapy with heme oxygenase- 1 limits infarct size 1 year later without adverse functional consequences. Basic Res Cardiol. 2011;106:1367-77.

47. Tang XL, Nakamura S, Li Q, Wysoczynski M, Gumpert AM, Wu WJ, Hunt G, Stowers H, Ou Q and Bolli R. Repeated Administrations of Cardiac Progenitor Cells Are Superior to a Single Administration of an Equivalent Cumulative Dose. J Am Heart Assoc. 2018;7. 
48. Tokita Y, Tang XL, Li Q, Wysoczynski M, Hong KU, Nakamura S, Wu WJ, Xie W, Li D, Hunt G, Ou Q, Stowers $\mathrm{H}$ and Bolli R. Repeated Administrations of Cardiac Progenitor Cells Are Markedly More Effective Than a Single Administration: A New Paradigm in Cell Therapy. Circ Res. 2016;119:635-51.

49. Bolli R, Tang XL, Sanganalmath SK, Rimoldi O, Mosna F, Abdel-Latif A, Jneid H, Rota M, Leri A and Kajstura J. Intracoronary delivery of autologous cardiac stem cells improves cardiac function in a porcine model of chronic ischemic cardiomyopathy. Circulation. 2013;128:122-31.

50. Swindle MM, Smith AC, and Hepburn BJ. Swine as models in experimental surgery. J Invest Surg. 1988;1:65-79.

51. Swindle MM, Horneffer PJ, Gardner TJ, Gott VL, Hall TS, Stuart RS, Baumgartner WA, Borkon AM, Galloway E, and Reitz BA. Anatomic and anesthetic considerations in experimental cardiopulmonary surgery in swine. Lab Anim Sci. 1986;36:357-61.

\section{Figures}



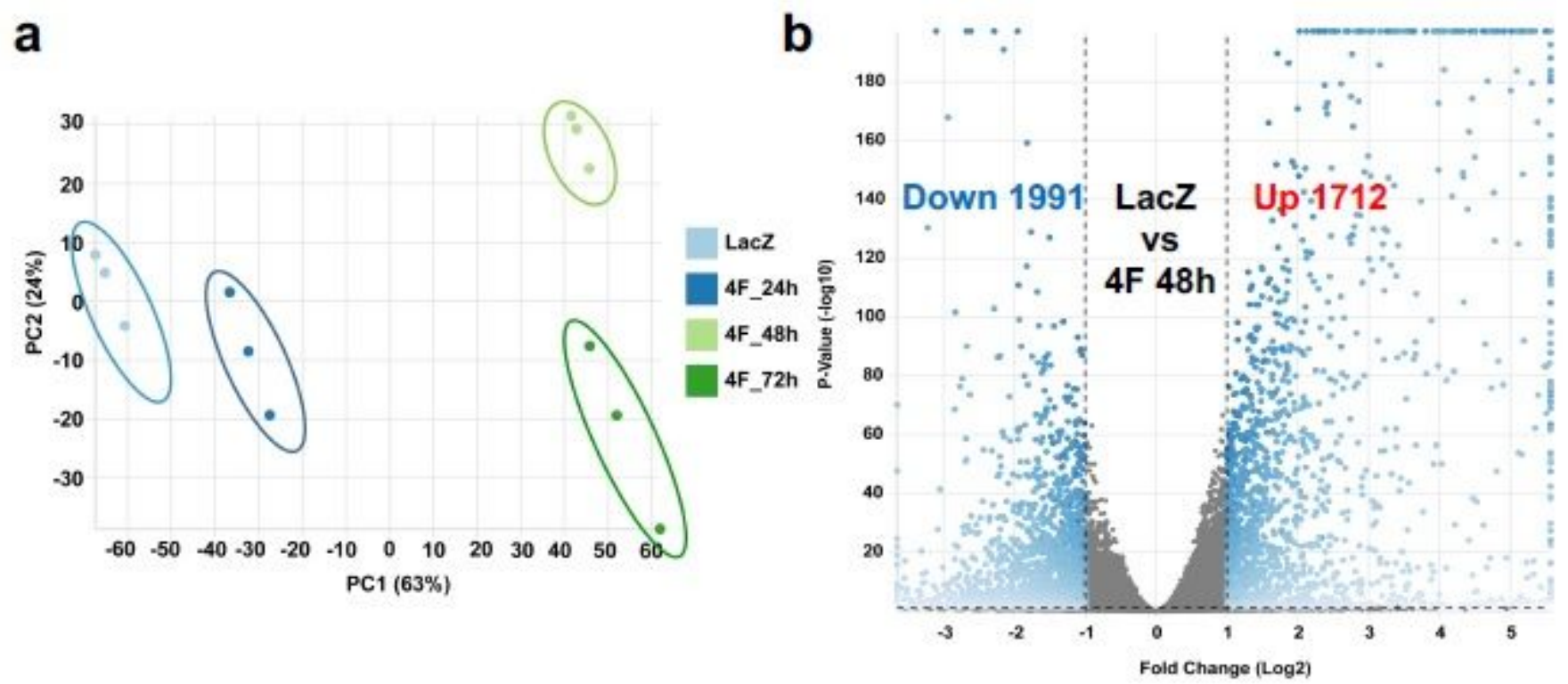

C

Upregulated GO terms in 4F group (48h)

d

NA strand elongation involved in DNA replication

regulation of cellular res ponse to heat

chromosome segregation

DNA synthes is involved in DNA repair

DNA replication initiation

G2/M transition of mitotic cell cycle

telomere maintenance via recombination

G1/S transition of mitotic cell cycle
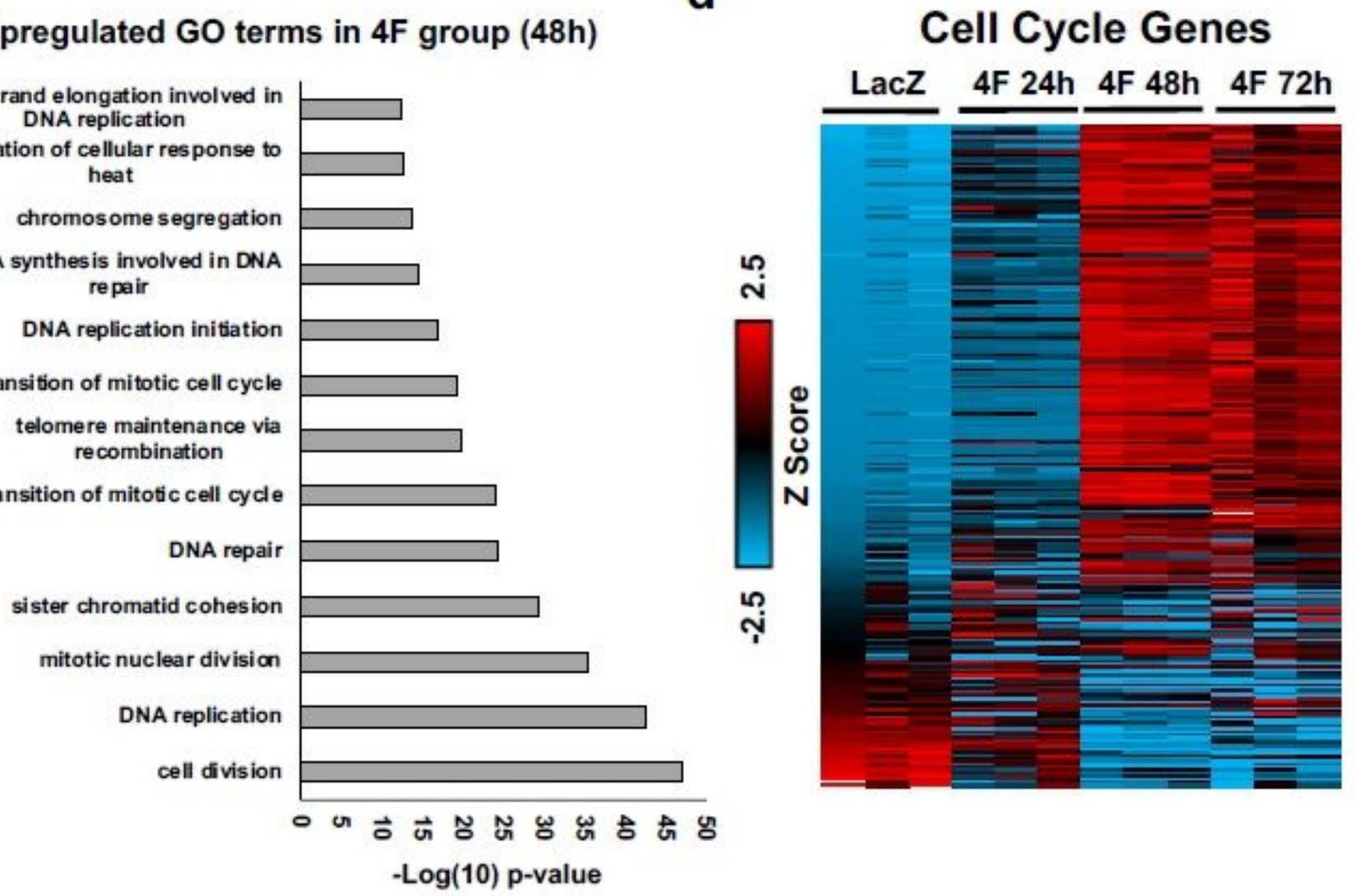

Cell Cycle Genes
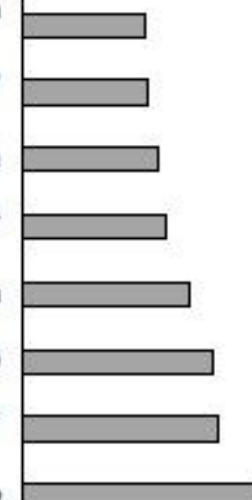

d

\section{Figure 1}

4F overexpression induces cell cycle reprogramming. (a) Principal component analysis (PCA) of RNA-seq data from mature hiPS-CMs (60 day old) infected with either LacZ (control) or $4 \mathrm{~F}$ for 24,48 , or $72 \mathrm{~h}$ ( $\mathrm{n}=$ 3). (b) Volcano plot demonstrating the number of genes that are significantly upregulated or downregulated $48 \mathrm{~h}$ post-4F adenovirus overexpression compared to the LacZ group. (c) The bar graph shows the top GO terms for the significantly upregulated genes from RNA-seq data comparing hiPS-CMs 
infected with either LacZ or 4F for $48 \mathrm{~h}$. The $\mathrm{GO}$ terms reflect mostly upregulation of cell cycle genes. (d) Row normalized Z score heatmap shows a temporal expression of over 200 genes related to cell cycle ( $n=3$ in each sample).
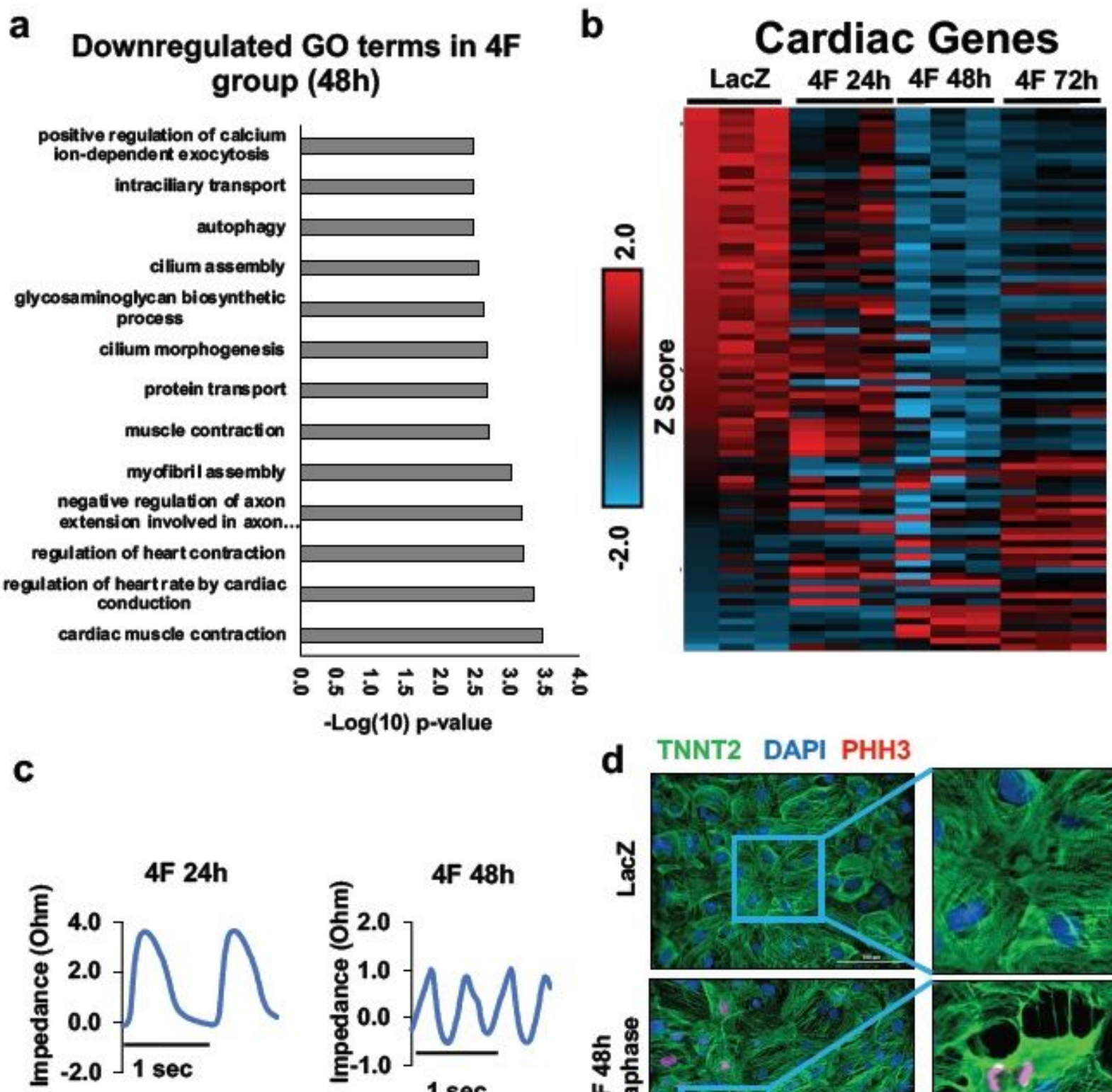

TNNT2 DAPI PHH3


Figure 2

Overexpression of $4 \mathrm{~F}$ for $48 \mathrm{~h}$ induces sarcomere disassembly. (a) The bar graph shows the top GO terms for the significantly downregulated genes from RNA-seq data comparing gene expression between the 
hiPS-CMs infected with either LacZ or $4 \mathrm{~F}$ for $48 \mathrm{~h}$. The $\mathrm{GO}$ terms reflect the downregulation of cardiac contractile and sarcomeric genes. (b) Row normalized Z score heatmap shows the contractile and sarcomeric gene expression in a time-dependent manner following $4 \mathrm{~F}$ expression ( $\mathrm{n}=3$ in each sample).

(c) Representative traces of impedance recording for contractile function from hiPS-CMs at 24, 48, and 84 $\mathrm{h}$ after infection with $4 \mathrm{~F}$. The bottom right panel shows the quantification of the contractile force amplitude comparing LacZ- and 4F-treated hiPS-CMs, 24,48 , and $84 \mathrm{~h}$ post-infection ( $\mathrm{n}=3$ independent experiments each in triplicate, ${ }^{\star} p<0.05$ compared to LacZ treated cells). (d) representative images of sarcomere disassembly during $\mathrm{G} 2 / \mathrm{M}$ phase in hiPS-CMs infected with lacZ (top panel), or 4F adenovirus for $48 \mathrm{~h}$ (middle (anaphase example) and bottom (cytokinesis example) panels) and stained with antibodies against the sarcomeric protein (troponin-T) (green), G2/M phase marker (PHH3) (red), and nuclear DAPI (blue). 

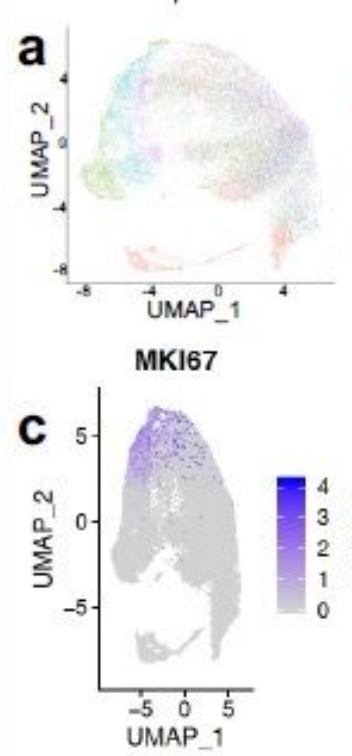

CDC20

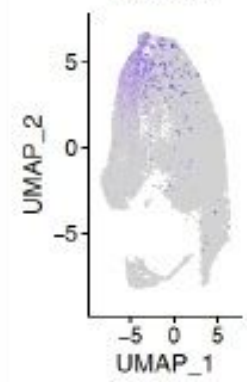

d

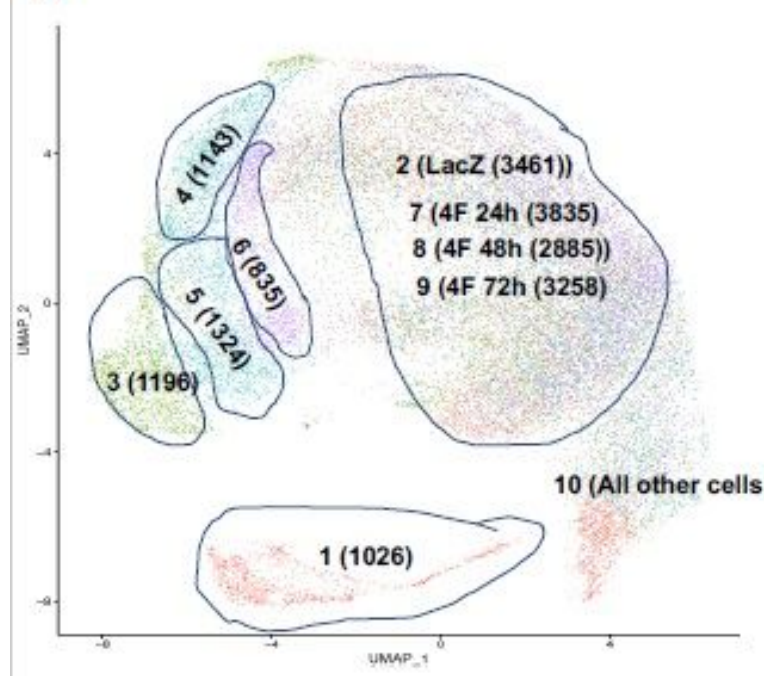

- LacZ (6761)

- 4F 24h (7006)

4F 48h (7269)

4F 72h (6294)

AURKB

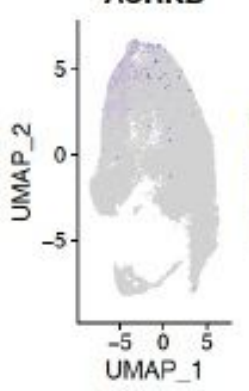

TK1

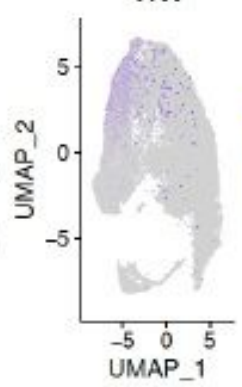

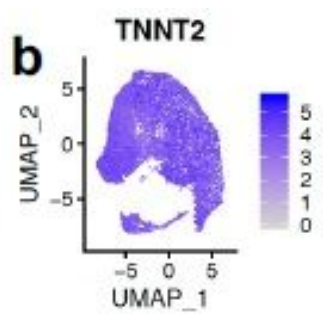

AURKA

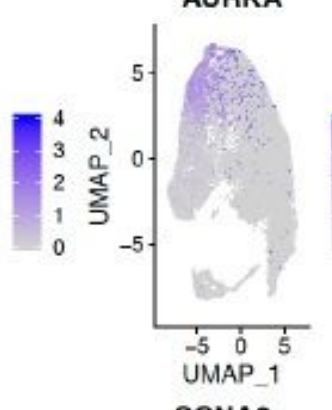

CCNA2

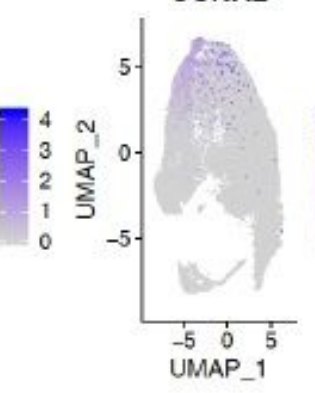

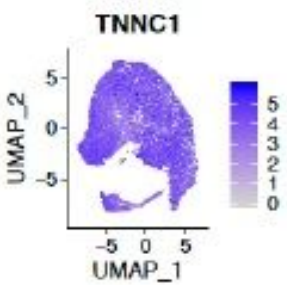

PCNA
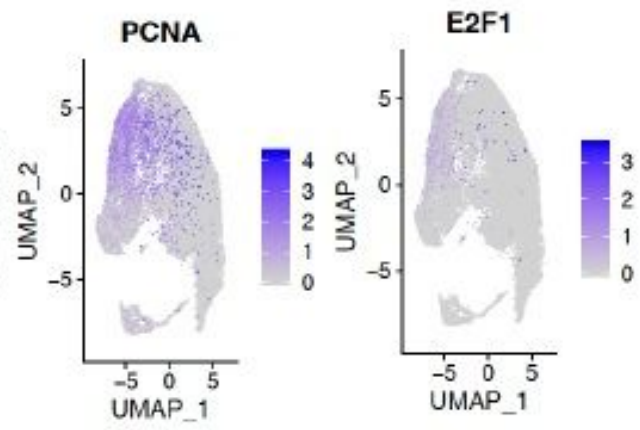

PLK1

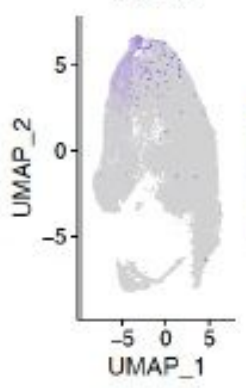

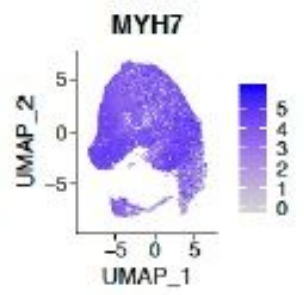

ANLN

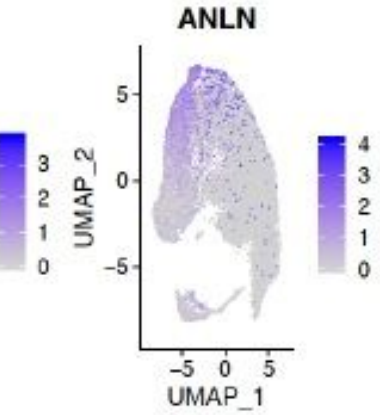

\section{Figure 3}

Single-cell RNAseq identifies a unique mitotic subpopulation that appears at $48 \mathrm{~h}$ post-infection with $4 \mathrm{~F}$. (a) UMAP plots considering global gene expression for all single cardiomyocytes sequenced from LacZ (control group) and 4F-overexpressing cardiomyocytes at $24,48 \mathrm{~h}$, or $72 \mathrm{~h}$ after viral transduction (approximately 7000 cells/sample). (b) All cells show high expression of the cardiac markers, TNNT2, TNNC1, and MYH7, indicating the cardiomyocytes' purity. (c) A unique cell population appears only at 48 
h post-infection with $4 \mathrm{~F}$ which express mitotic/cytokinesis genes; this population was identified as the mitotic population that expresses high levels of the G2/M markers (Ki67, Aurora Kinase A and B, P.C.N.A., E2F1, CDC20, TK1, CCNA2, PLK1, and A.N.L.N.). (d) re-clustering the cells according to their expression of the mitotic genes. Consistent with our published data, the mitotic population represents $\sim 15 \%$ of the total cardiomyocyte population within the $48 \mathrm{~h}$ sample. (d) Trajectory analysis using MONOCLE algorithm matching the logical time course of the unique populations at each time point.

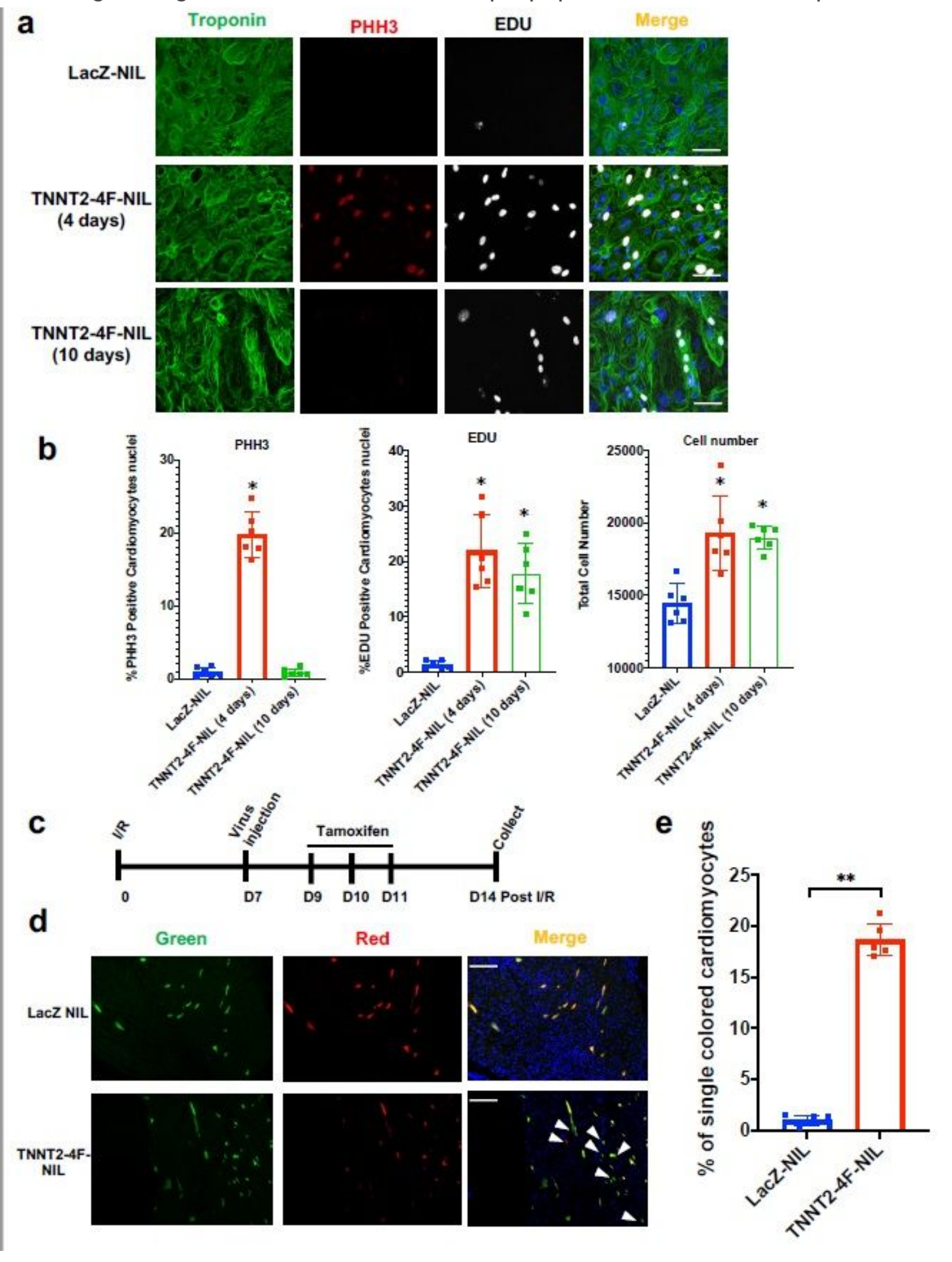

Figure 4 
Transient expression of the $4 \mathrm{~F}$ using non-integrating lentivirus (NIL) is efficient in inducing cardiomyocyte proliferation in vitro and in vivo. (a) Representative images of hiPS-CMs treated with LacZ-NIL, TNNT2-4FNIL for 4 or 10 days, and immuno-stained for troponin-T (green), PHH3 (red), EDU (gray) (scale bar=100 $\mu \mathrm{m})$. (b) Quantification of percentage increase in proliferation markers (PHH3 and EDU) and total cell number/well ( $n=6$ independent experiments conducted in duplicate, ${ }^{*} P<0.05$ vs. LacZ NIL). (c) Schematic diagram of the experimental design for MADM mice injection with the TNNT2-4F-NIL or LacZ-NIL control. (d) Representative images show single-colored cardiomyocytes of MADM mice hearts treated with LacZNIL or TNNT2-4F-NIL (Scale bar=100 $\mu \mathrm{m}$ ). (e) Quantification of the percentage of the single-colored cells to the total labeled cells ( $n=6$ animals per group, ${ }^{\star *} p<0.01$ vs. LacZ NIL). 


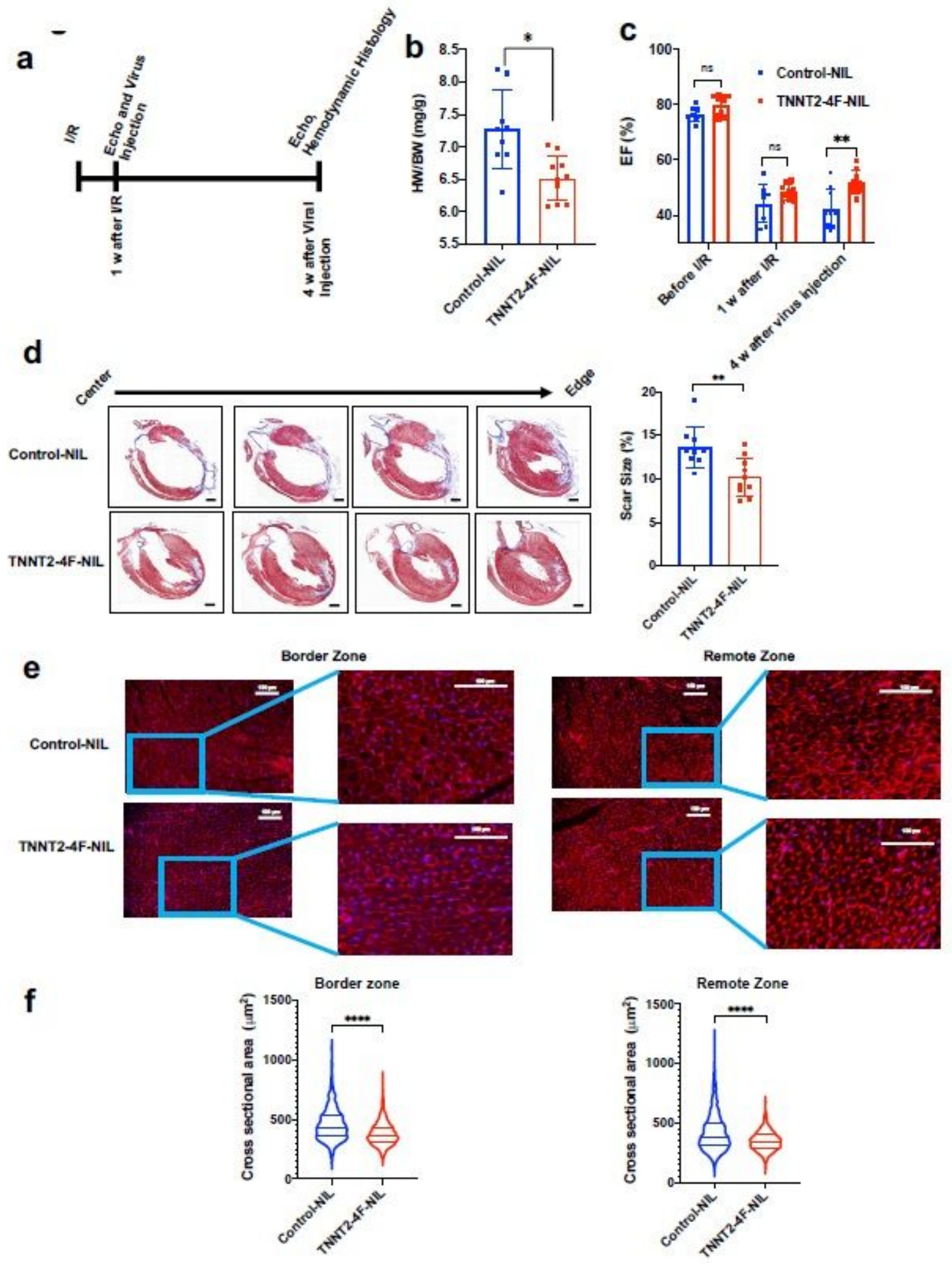

\section{Figure 5}

Transient expression of the 4F using TNNT2-4F-NIL improves cardiac function and reduces scar size in rats after I/R. (a) schematic diagram of the experimental design. (b) Quantification of heart weight/ body weight ratio (HW/BW). ( $n=9-10$ rats per group, ${ }^{*}<<0.05$ compared to GFP-NIL control group) (c) Ejection fraction (EF), as assessed by echocardiography before Ischemia/reperfusion (I/R), one week after I/R (before viral treatment), and four weeks after viral treatment. ( $n=9-10$ rats per group, ${ }^{\star} p<0.05,{ }^{* \star} p<0.01$ 
compared to GFP-NIL control group) (d) Representative images of rat hearts stained with Masson's trichrome stain (right panel) (healthy myocardium stains red and fibrotic tissue stains blue) at the end of the experiment (scale bar $=2 \mathrm{~mm}$ ). The left panel shows scar size quantification as a percentage of total heart tissue ( $n=9-10$ rats per group, 20-25 heart sections per animal, ${ }^{* *} p<0.01$ compared to the control group). (e) Representative images of rat heart at the border zone (right panel) or a remote zone (left panel) stained against wheat germ agglutinin, WGA, (red), and nuclear DAPI (blue) (scale bar=100 $\mu \mathrm{m}$ ). (f) Quantification of the cross-sectional area of cardiomyocytes at border zone (left) or a remote zone (right) ( $n=1300-1500$ cells from each group, 20-25 heart sections per animal ${ }^{\star \star \star \star} p<0.0001$ compared to control group). 


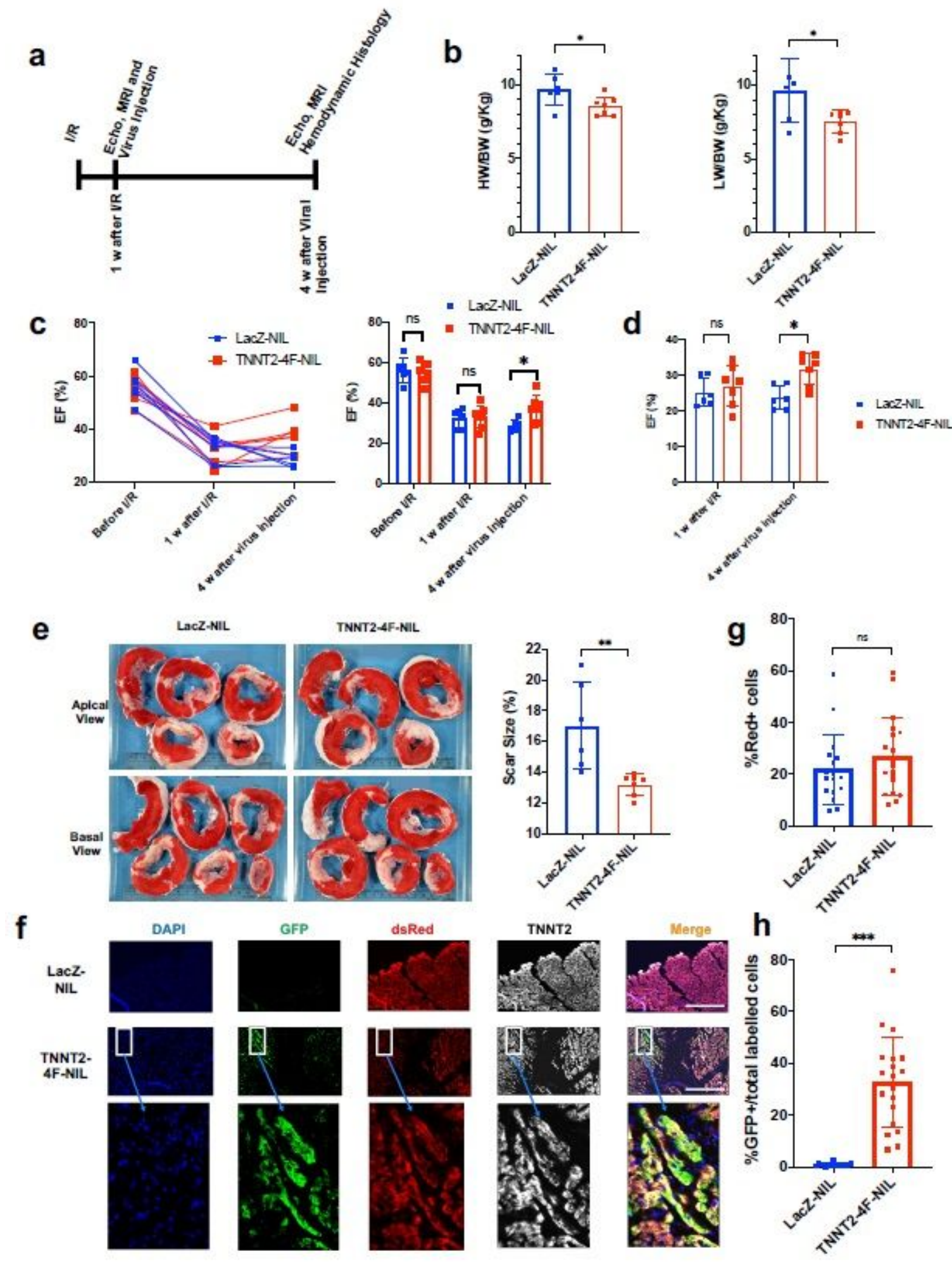

Figure 6

Transient expression of the 4F using TNNT2-4Fpolycistronic-NIL improves cardiac function, reduces scar size, and induces cardiomyocyte proliferation in a porcine model of heart failure. (a) schematic diagram of the experimental design. (b) Quantification of heart weight/body weight ratio (HW/BW) (left panel), lung weight/body weight ratio (LW/BW) (right panel). ( $n=6-7$ pigs per group, ${ }^{*} p<0.05$ vs. control LacZ group) (c) Quantification of ejection fraction (EF) as assessed by echocardiography in individual pigs 
(left panel) before Ischemia/reperfusion (I/R), one week after I/R (before viral treatment), and four weeks after viral treatment. The right panel shows the average ejection fraction for all pigs $(n=6-7$ pigs per group, ${ }^{*} p<0.05$ vs. control LacZ group). (d) Quantification of average ejection fraction (EF) as assessed by MRI 1 week after I/R (before viral treatment) and four weeks after viral treatment ( $n=6-7$ pigs per group, ${ }^{*} p<0.05$ vs. control LacZ group). (e) Left panel, representative images of pig hearts stained with triphenyl tetrazolium chloride (T.T.C.) (healthy myocardium stains red and fibrotic tissue remains white) at the end of the experiment (scale bar in $\mathrm{cm}$ ). Right panel, quantification of scar size as a percentage of total heart tissue ( $n=6-7$ pigs per group, ${ }^{\star \star} p<0.01$ vs. control LacZ group). (f) Representative images at the site of injection four weeks post-injection in porcine myocardium. LacZ (top panel) or 4F (bottom panel) shows the expression of the proliferation double reporter system GFP and dsRed and their co-localization with cardiomyocytes (TNNT2). (g) Quantification of the percentage of dsRed positive cardiomyocytes and (h) GFP positive cardiomyocytes ( $n=6-7$ pigs per group, $3-4$ sections from different injection sites ${ }^{\star \star \star} p<0.001$ vs. control LacZ group).

\section{Supplementary Files}

This is a list of supplementary files associated with this preprint. Click to download.

- Supplementarymovie1LacZAurKBGFP.mov

- Supplementarymovie24FAurKBGFPConverted.mov

- Supplementarymovie3LacZ4Wafterviralinjection4chamber.mov

- Supplementarymovie4LacZ4Wafterviralinjectionshortax.mov

- Supplementarymovie54F4Wafterviralinjection4chamber.mov

- Supplementarymovie64F4Wafterviralinjectionshortax.mov

- supplementaryfigure.pdf 